\title{
Über den gemeinsamen Sprachgebrauch in Ost und West, seine Probleme und kreativen Möglichkeiten
}

\begin{abstract}
In dieser Arbeit über den neueren deutschen Sprachgebrauch in Ost und West soll nicht das Trennende betont werden, sondern das gemeinsame Spracherbe und seine Wirkung in der Gegenwart. Aber eine Beobachtung gegenüber vielen kritischen Stimmen aus West und Ost sei, in völlig unpolemischer Absicht, nicht verschwiegen: $\mathrm{Zu}$ viele sprachkritische Artikel aus der Bundesrepublik haben in der Vergangenheit von der aus Texten bekannten offiziellen Sprache der DDR-Machthaber zu direkt auf die wirklich gesprochene Sprache der Bevölkerungsmehrheit geschlossen, und zu viele DDR-Sprachwissenschaftler und Polemiker haben auf die westliche Kritik vor allem als Verteidiger des politischen Kurses der SED reagiert, aus welchen persönlichen Gründen auch immer. Gedruckt worden wären andere Meinungen in der DDR allerdings nicht, und der Karriere waren sie auch nicht förderlich. Trotzdem gilt: Die deutsche Sprache hat sich in Ost und West auf jeweils spezifische Weise weiterentwickelt, sie wurde aber nicht gespalten und funktionierte in Ost und West und - trotz spürbarer Unterschiede - zwischen Ost und West als gemeinsames Verständigungsmittel, wo immer sie dafür benutzt wurde. Zwischen den Begriffen „Sprachwandel“ und „Bezeichnungswandel“" muss sorgfältig unterschieden werden. Im kreativen Umgang mit dem gemeinsamen Deutsch haben sich Ost und West längst wieder zusammengefunden. Genauere Untersuchungen über das Alltagsdeutsch in der DDR sind sehr zu wünschen, die Schaffung eines brauchbaren Korpus dafür ist im Nachhinein nicht einfach, sollte aber in Angriff genommen werden.

This article on recent German linguistic usage in east and west stresses not the differences, but the common linguistic heritage and its effect in the present. However, one observation on many critical views from both west and east may be allowed, without any polemical intention: Too many articles on this issue from the Federal Republic have in the past made a direct connection between the official language contained in texts published by the GDR ruling elite and the genuine spoken language of the majority of the population, and too many GDR linguists and polemicists have reacted to western criticism first and foremost as defenders of the political course of the SED, for whatever personal reasons. It must be acknowledged, of course, that other views would not have been published in the GDR and that they would not have been conducive to people's careers. Nevertheless it is true that the German language developed in specific ways in east and west, but that it was not split and functioned in east and west and as a common means of communication wherever it was needed - in spite of noticeable differences between east and west. The terms "linguistic change" and "change of designation" must be carefully differentiated. East and west have long since come together in the creative use of the common German language. More precise studies of the everyday German in the GDR would be very desirable, and although the creation of a useable corpus of this variety after the event would not be easy, it should be undertaken.
\end{abstract}

\section{Vorbemerkungen}

Deutschland feiert den 20. Jahrestag der Wiedervereinigung oder, verfassungsjuristisch korrekter, das Jubiläum des Beitritts der DDR zur Bundesrepublik Deutschland, ganz sicher aber den zwanzigsten Jahrestag seiner sprachlichen Wiedervereinigung. Während der vergangenen mehr als sechzig Jahre sind vor allem die negativen Folgen der Trennung immer wieder kritisch beobachtet und auf beiden Seiten linguistisch und politisch interpretiert und kommentiert worden. Das waren die Zeiten der Sowjetischen oder Russischen Besatzungszone, der Ostzone oder einfach der ,Zone“ und dann der DDR auf der einen 
Seite und der Amerikanischen, Britischen, Französischen Zone, der Bizone und Trizone und eben der Bundesrepublik Deutschland auf der anderen Seite. Für die westliche Kritik am deutschen Osten spielte dessen schwierige Situation unter dem maßgeblichen politischen und wirtschaftlichen Einfluss der Sowjetunion eine entscheidende Rolle. Die Beteiligung am Marshallplan, der Grundlage des „Wirtschaftswunders“, musste in Ostdeutschland abgelehnt werden (ich erinnere mich noch lebhaft an die heftigen Streitgespräche darüber in der Schulzeit in meiner Heimatstadt Frankfurt an der Oder). In Westdeutschland wurde die enge Anbindung an die Westmächte politisch ermöglicht und wirtschaftlich belohnt. Das prägte das Selbstbewusstsein auch der westlichen Sprachkritiker, während ihre östlichen Antipoden die Propaganda der dort herrschenden Partei nur recht und schlecht zu verteidigen wussten. Wer sich über die alten Debatten um Russifizierung oder Amerikanisierung des Nachkriegsdeutschen und die genauere und modernere Kritik an Wortschatzentwicklungen oder Kommunikationsdifferenzen informieren will, verschaffe sich sein eigenes Urteil durch die Lektüre der neueren Fachliteratur. ${ }^{1}$

Der Mehrzahl der westlichen Kritiker der deutschen Sprache in der DDR hat ganz sicher die intime Einsicht in das Sprachverhalten der östlichen „Brüder und Schwestern“ gefehlt (und fehlen müssen), wirklich gekannt haben die meisten nur die offizielle Sprache der DDR, also die der durch die SED verantworteten und gelenkten Medien (Zeitungen, Fernsehen, Radiosender) und der Zeitungen der in allen wesentlichen politischen Fragen gleichgeschalteten sonstigen Parteien und gesellschaftlichen Organisationen (Freier Deutscher Gewerkschaftsbund [FDGB], Freie Deutsche Jugend [FDJ], Demokratischer Frauenbund Deutschlands [DFD], Kulturbund zur demokratischen Erneuerung Deutschlands [KB, seit 1974 ,Kulturbund der DDR“]). Nur die SED und die Vertreter der von ihr abhängigen Parteien, einschließlich der eben genannten Organisationen, waren in der Volkskammer vertreten und besaßen die Macht über die gedruckten politischen Texte. Die einzigen gesellschaftlichen Organisationen, die der Kontrolle und Einflussnahme durch die SED nennenswerten Widerstand leisten konnten, waren die evangelische und katholische Kirche, andere kirchliche Konfessionen und die kleinen jüdischen Gemeinden. Fast alle verfügten auch sie über eigene, frei abonnierbare Wochen- oder Monatszeitungen und konnten an vielen großen und kleineren Orten auch eigene Kindergärten betreiben, also das angestrebte Erziehungsmonopol des Staates unterlaufen. Die Sprache der kirchlichen Presse und die - sicher bescheidene - Bedeutung der Kirchen für die Sprachbildung der DDR ist meines Wissens bisher aber nie thematisiert worden.

Horst-Dieter Schlosser hat noch 1990 angemerkt, es werde ,oft ohne weitere Reflexion angenommen, dass die öffentliche, d. h. noch genauer: die veröffentlichte Sprache nicht nur politisch, sondern auch sachlich für die Kommunikation einer Sprachgemeinschaft repräsentativ sei" (Schlosser 1990, S. 157). Der direkte Schluss von der offiziellen Sprache auf die wirklich gesprochene greift aber gerade in autoritär und repressiv organisierten

1 Das sprachgeschichtliche Grundlagenwerk von v. Polenz (1999) gibt sachlich und fair Auskunft über die sprachlichen Entwicklungen in den beiden deutschen Nachkriegsstaaten. Konzentriert und lesenswert ist die Sammelrezension von Burkhard Schaeder (2009) über Manfred W. Hellmann/Marianne Schröder (Hg.), Sprache und Kommunikation in Deutschland Ost und West. Ein Reader zu fünfzig Jahren Forschung. Hildesheim 2008, sowie Manfred W. Hellmann, Das einigende Band? Beiträge zum sprachlichen Ost-West-Problem im geteilten und wiedervereinigten Deutschland. Hg. von Dieter Herberg. Tübingen 2008. Vgl. außerdem: Schlosser (1999); Schmidt (2000 b), alle Titel mit reichen Literaturangaben. Über die ältere einschlägige Literatur informierte früh und sorgfältig Hellmann (1976). 
Gesellschaften fast immer viel zu kurz. Zwar dringt aus diesen Gesellschaften auf den offiziellen Wegen in der Regel eben nur die obrigkeitlich organisierte und kontrollierte veröffentlichte Sprache ohne Schwierigkeiten nach außen, aber zwischen der Alltagssprache der Mehrheit und der gelenkten Sprache des Regimes pflegt es erhebliche Differenzen zu geben (in der Regel mit mehreren funktional bedingten Vermittlungsstufen zwischen Alltags- und offizieller Sprache). Schlosser urteilt über frühere, zu undifferenzierte Arbeiten zur Sprachverwendung in der DDR:

\footnotetext{
„Die Ausblendung der alltäglichen und privaten Kommunikation vor der DDR-Oktoberrevolution war indes weniger ein Defizit an gutem Willen der westlichen Sprachbeobachter, weil sie durch die administrative Verhinderung empirischer Forschungen in der DDR erzwungen war. Gleichwohl haben nicht wenige Darstellungen der Sprachverhältnisse in der DDR aus der Not eine Tugend gemacht und vielfach den Eindruck erweckt, dass die Sprache der Herrschenden in der DDR die Sprache der Bevölkerung schlechthin gewesen sei.“ (Schlosser 1990, S. 194)
}

Einen solchen Eindruck erweckten manchmal schon die Titelformulierungen bundesdeutscher Beiträge, so wenn eine durchaus lesenswerte Arbeit auf dem Titelblatt formulierte: „Der Deutschlandbegriff der DDR. Dargestellt vornehmlich an der Sprache des Neuen Deutschland“ (Marzahn 1979, S. 3). Offenbar hat erst das Jahr 1989 den zahlreichen an der Sprache interessierten, aber am Verstand ihrer Brüder und Schwestern zweifelnden Beobachtern gezeigt, dass der „Deutschlandbegriff der DDR" eben keineswegs einheitlich, sondern (wie viele andere auch) nicht auf eine einfache Formel zu bringen war, schon gar nicht auf die der SED. Die Differenzierung zwischen offizieller Sprache und Alltagssprache, zwischen offiziellem Denkschema und dem wirklichen, „bunten“ Denken der lebendigen Mehrheit war und ist erforderlich, denn die Texte des Neuen Deutschland entsprachen bestenfalls den Überzeugungen der Mehrheit seiner Leser. Das Vertrauen darauf, dass die DDR-Bürger dem Druck ihres Regimes in nennenswerter Zahl den eigenen Kopf entgegensetzen würden, also die Hoheit über ihren Sprachgebrauch nicht ohne weiteres an das Neue Deutschland abtreten würden, war bei vielen westlichen Sprachbeobachtern nicht sehr ausgeprägt. Eine noch recht gemäßigte Stellungnahme sei zitiert. Unter Verweis auf das Deutsch der ersten Befehle der Sowjetischen Militärverwaltung meinte Ernst Riemschneider: „Auf diese Weise war die Bevölkerung [...] in zunehmendem Maße der Sowjetterminologie ausgesetzt. In der gegenwärtigen Lage ist es schwierig zu untersuchen, wieweit die Umgangssprache durch diese Terminologie beeinflusst wurde" (Riemschneider 1963, S. 8). Der Autor begrüßte deshalb die Herausgabe des Wörterbuchs der deutschen Gegenwartssprache (WDG) durch die Deutsche Akademie der Wissenschaften in Berlin, weil dieses Wörterbuch ,möglicherweise der westlichen Welt sehr dienlich sei““ für die Beobachtung der ,deutschen Sprache in der sowjetisch besetzten Zone Deutschlands" (so in Riemschneiders Titelformulierung), deren ,Veränderungen bereits sehr weit fortgeschritten sind" (Riemschneider 1963, S. 11). Ähnliche Sorgen bewegten damals Heinrich Scholz, aber er differenzierte zwischen Schriftsprache und Alltagssprache: „Mag bei der Analyse der deutschen Schriftsprache der Zweifel auftauchen, ob wir überhaupt noch eine deutsche Sprache sprechen, so weist die Sprache des Alltags viel geringere Unterschiede zwischen hüben und drüben auf" (Scholz 1964, S. 92). Allerdings vermischte Scholz seinen Begriff der Schriftsprache offenbar mit dem des gedruckten „Parteijargon" (so ebenfalls S. 92). Der Schriftsprache haben sich in der DDR aber nicht nur die Anhänger der SED bedient, und der Blick in die Sprache der DDR-Literatur darf gewiss nicht mit dem in das Neue Deutschland gleichgesetzt oder verwechselt werden. Natürlich 
war der Wunsch nach intimerer Kenntnis der wirklich gesprochenen Alltagssprache der DDR auf der Grundlage der Texte östlicher Zeitungen schwer zu erfüllen, aber sogar noch nach dem Mauerbau hätten interessierte Sprachforscher trotz aller Schwierigkeiten der Annäherung an den Osten engere Bekanntschaft mit der tatsächlich gesprochenen Sprache etwa in Berlin schließen können. Romanisten, die sich für Kreolsprachen in der Karibik interessierten, hatten es gewiss schwerer. Riemschneider trieb im Übrigen schon 1963, nur fünfzehn Jahre nach der Etablierung zweier deutscher Staaten, die Sorge um, die sprachlichen Sonderentwicklungen könnten noch zunehmen: „Falls dem so ist, kann es eines Tages geschehen, dass die Vertreter Westdeutschlands und Sowjetdeutschlands einen Dolmetscher benötigen, wenn sie sich am Konferenztisch treffen" (Riemschneider 1963, S. 5). Heute, zwanzig Jahre nach dem Tanz auf der Mauer, dürfen wir froh sein, dass wir Dolmetscher nie gebraucht haben. Walther Dieckmann hat die Sprachwissenschaftler mit sozialwissenschaftlichen Argumenten schon 1969 gewarnt, „die sprachlichen Wirkungen der Propaganda“ und die „Macht der Sprache zu hoch einzuschätzen“, weil „die Beeinflussungsmöglichkeiten des Denkens und Verhaltens durch die Propaganda recht begrenzt sind" (Dieckmann 1969, S. 117). Dieckmann hat recht behalten. Jede Propaganda, die mit wirklichkeitsfernen Floskeln argumentiert, wird irgendwann an der Realität scheitern. Nun stellt sich natürlich die Frage, wie wollen wir denn heute noch beweisen, dass unsere Behauptung stimmt, die Alltagssprache der Mehrheit der DDR-Bürger sei nicht identisch mit der offiziellen und Propagandasprache der SED. Das wäre eine Aufgabe für Sprachwissenschaftler und Informatiker: Wir müssen versuchen, Texte zu definieren und in Korpora zusammenzufassen, die als Zeugnisse dieser Alltagssprache gelten können, auch wenn vermutlich in vielen Einzelfällen Abstriche vom Erfassungsideal zu machen wären. Heranzuziehen und zu prüfen wären vor allem private Texte, also Briefe und Tagebücher aus der DDR, aber durchaus auch literarisch gefasste alltagssprachliche Passagen in Werken von DDR-Schriftstellern innerhalb und außerhalb der ehemaligen Grenzen dieses Staates. Es ist ja nicht so, als hätten gute Schriftsteller wie Günter de Bruyn, Ulrich Plenzdorf, Brigitte Reimann, Christa Wolf (und so viele andere, bekannte und unbekannte) in ihren Texten immer nur die Sprache der SED gebraucht oder ihre Figuren nur diese Sprache reden lassen. Natürlich werden dabei auch Abstufungen der Annäherung an die offizielle Sprache deutlich werden, aber das wird sich zeigen, und das macht ja die Zusammenstellung und Benutzung eines solchen Materials spannend und wichtig. Für die Schwierigkeiten nur ein Beispiel: Belege für den Ausdruck 150prozentige als substantivische Personenbezeichnung sind verständlicherweise in den Medien der DDR kaum zu erwarten (oder höchstens als Beleidigungstatbestände). DWDS und COSMAS II bieten keine Nachweise für den vollkommen üblichen umgangssprachlichen DDR-Gebrauch vor 1989. Den frühesten DDR-spezifischen Beleg bietet COSMAS II aus der TAZ am 13.10.1989. Erst in ,indigenen" Erinnerungstexten finden sich gelegentlich Belege wie: die Parteisekretärin [...] ein berïchtigter Drache, eine 150-Prozentige (Birgit Walter in: BERL. ZTG. 11./12.7.2009, S. 8).

Zwar haben die 68er in der Bundesrepublik gemeint, es gebe kein richtiges Leben im falschen, aber diese These entspricht nicht der Lebenserfahrung eines sehr großen Teils der DDR-Bürger, die sich im falschen Gesellschaftssystem gegen alle Schwierigkeiten, gemeinsam mit ihren Familien und Freunden, um ein möglichst richtiges Leben ernsthaft bemüht haben. Und genau dazu gehörte notwendig eine ausreichende kritische Distanz zur Sprache des Regimes. Die Sprachwissenschaft kennt längst die Unterscheidung von 
passiver und aktiver Kompetenz. Wir alle üben unsere passive Kompetenz im Wortverstehen als Hörer und Leser für einen weit umfangreicheren Wortschatz, als wir in unserer Rede- und Schreibpraxis selbst aktiv realisieren. So hat sich auch der DDR-Bürger das für seine Lebenswirklichkeit notwendige Verständnis für die Besonderheiten der offiziellen Sprache der DDR zumindest passiv angeeignet, aber im eigenen aktiven Gebrauch dieser Besonderheiten, also in der selbst gesteuerten Performanz (bzw. in der Ausübung ihrer kommunikativen Kompetenz im Sinne von Dell Hymes) haben sich viele sehr zurückgehalten. Vor allem im privaten Bereich unterlag der Gebrauch dieses Wortschatzes der Entscheidung jedes Einzelnen. Namen von Institutionen und Benennungen von Aktivitäten, die auch im privaten Bereich besprochen werden mussten, wurden natürlich benutzt, ideologisch geprägte Begriffe der offiziellen Sprache dagegen dort eher nicht. Umgekehrt war der besondere Wortschatz der Bundesrepublik, soweit er in den westlichen Radio- und Fernsehsendern benutzt wurde und die eigenen Interessen der DDR-Bürger berührte, den meisten nicht nur gut bekannt, sondern in vertrauter Umgebung auch in aktivem Gebrauch. Diese Sender konnten in den letzten Jahrzehnten der Teilung fast überall ungestört gehört werden. Sie verstärkten nicht nur die Bindung an das gemeinsame Deutsch in Ost und West, sie verbreiteten auch die Kenntnis von lexikalischen Neubildungen und besonderen Gebrauchsweisen aus der BRD. Die umgekehrte Wirkung der DDR-Sender war naturgemäß wesentlich geringer. Das DDR-Publikum der westlichen Sender bestand keineswegs nur aus überzeugten Antikommunisten, sondern rekrutierte sich sehr weitgehend aus allen Teilen der DDR-Gesellschaft, sogar aus den Mitgliedern und der ideologischen Klientel der SED. Nach der Wende verbreitete sich zwar die Ansicht, die DDR-Bürger verstünden die Sprache des Westens nicht und die Bundesbürger nicht die der DDR. Alle möglichen Probleme des Zusammenwachsens wurden auf die sprachliche Entfremdung zurückgeführt. Sehr oft zu Unrecht. Ungünstig für ein besseres Verstehen war allerdings der (berechtigte oder unberechtigte) Eindruck vieler DDR-Bürger, ihnen sollte nach der ersten Euphorie über die wiedergewonnene Einheit als eigene Schuld die Verantwortung für die Spaltungspolitik der Sowjetunion und der SED-Führung und damit für das Versagen der DDR auf vielen Gebieten angelastet werden. Dagegen half natürlich auch keine Erinnerung an die schon unter Adenauer angestrebte und erreichte stabile Westbindung der Bundesrepublik und das damit verbundene bewusste Inkaufnehmen einer dauernden Abhängigkeit des Ostens von der Sowjetunion, weil Adenauer das politische Risiko der Anbindung des unsicheren deutschen Ostteils zu groß erschien.

Die These einer ernsthaften sprachlichen Entfremdung als Ursache mangelhafter Verständigung ist in der Tat nur sehr eingeschränkt korrekt. Sie traf auf östlicher Seite am ehesten zu auf die im Ost- und West-Sprachgebrauch abweichenden Teile der Fachwortschätze der Verwaltung, der Wirtschaft, des Rechtswesens und einiger kleinerer Bereiche (einschließlich einer Reihe divergierender Fremdwortanteile). Die Kenntnis oder Nichtkenntnis bestimmter Fachwortschätze entscheidet aber kaum je über Spracheinheit oder Sprachspaltung. Derartige Unterschiede sind seit langem viel ausgeprägter zwischen dem Deutsch in Österreich, in der Schweiz und in Deutschland (Ost wie West), ohne geradezu systematisch zu entsprechenden ernsthaften Klagen über die Verschiedenheit der Sprachvarianten geführt und ohne je die Wirkung der deutschen Literatur als des einigenden Bandes über alle Staatsgrenzen hinweg oder die Kommunikation zwischen Deutschen, Österreichern und Schweizern (beim Gebrauch der Schriftsprache) in Frage gestellt zu haben. Solange die Kenntnis des Kernwortschatzes, der Grammatik und der wichtigsten Kommunikati- 
onsregeln gemeinsamer Besitz einer Sprachgemeinschaft ist, lassen sich solche Differenzen leicht kompensieren und schnell neutralisieren. Damit soll der Erfahrung nicht widersprochen werden, dass bestimmte sprachliche Defizite der ehemaligen DDR-Bürger ihnen bei der Eingewöhnung in die nun westlich geprägte wirtschaftliche und politische neue Einheit in den Wendejahren schmerzlich bewusst geworden sind, zumal sie auf der anderen Seite des Öfteren nicht nur Unverständnis, sondern Mitleid oder Hohn und Spott ausgelöst haben (bis hin zur politisch motivierten Rüge „falschen“ östlichen Sprachgebrauchs im Bundestag). Fremdheitsgefühle der neuen Bundesbürger wurden aber vermutlich weniger durch unüberwundene Sprachprobleme geweckt und genährt als durch die Ablösung der einigermaßen vertrauten DDR-Bürokratie durch die zunächst viel komplizierter erscheinende der Bundesrepublik in Rechts-, Steuer-, Wirtschafts- und einigen anderen Lebensfragen: Man verstand sich nicht (und wollte sich wohl manchmal auch nicht verstehen), weil man die neuen bürokratischen Verfahren im Osten überwiegend als lästig erlebte. Richter, Anwälte, Beamte, Steuerberater und neue Kollegen oder Westkonkurrenten durften sich da sehr überlegen fühlen und zeigten das nicht ganz selten auch. Das erzeugte Ärger und Frust, der von beiden Seiten gern oder höflicherweise auf sprachliche Ursachen geschoben wurde.

Man muss aber den Vergleich der Sprachverständlichkeit auf der innerdeutschen OstWest-Achse mit den Verhältnissen zu den Schweizer und österreichischen Nachbarn gar nicht bemühen, denn ein Blick auf die Verständigung zwischen Sprechern ausgeprägter regionaler Varianten der gemeinsamen Schriftsprache zeigt uns, dass die durch die deutsche Teilung verursachten Differenzen weit hinter denen zwischen einigen regionalen Varianten der Hoch- und Schriftsprache zurückstehen. Wird in Landschaften mit lebendigem Dialektgebrauch tatsächlich Dialekt gesprochen (z. B. im Fernsehen, wenn hier auch meist in gemäßigter Form), so versteht der Fremde fast nichts, und sogar der Gebrauch der regional geprägten Hochsprache durch die Einheimischen gibt ihm noch manche Rätsel auf. Dabei sind die Verständnisschwierigkeiten etwa zwischen Nieder- und Oberbayern oder auch Schwaben auf der einen Seite und Mittel- und Norddeutschen auf der anderen erfahrungsgemäß auch bei beidseitigem Gebrauch ihrer regional geprägten Hochsprache sicherlich größer als die zwischen Ost- und Westdeutschen aus Mittel- oder Norddeutschland. Aber Unterhaltungen mit Bayern und Schwaben machen Freude und unterliegen nicht dem Verdikt der Sprachspaltung, das uns im Ost-West-Verkehr so viele Sorgen bereitet.

Das größte Hindernis für die Untersuchung des wirklichen Sprachgebrauchs der DDRBevölkerung, also nicht der offiziellen Sprache der Parteipropaganda, der Sprache der Grenz- und Volkspolizisten und der Behörden, war die Praxis der Abschottungspolitik und deren Auswirkungen auf die Kontaktbereitschaft in Ost und West. Wer in den Jahrzehnten der Spaltung den wirklichen nicht-öffentlichen Sprachgebrauch der DDR-Bevölkerung kennen lernen wollte, brauchte vielfältige, zeitlich aufwendige Kontakte und das Vertrauen seiner Gesprächspartner. Dazu gehörten auf beiden Seiten ganz offenbar ungewöhnlicher Mut, Risikobereitschaft und ein ernstes Interesse am Leben der anderen. Diese Hürden haben vor 1990, aber auch noch Jahre danach, nur wenige Sprachkritiker mit Erfolg übersprungen. ${ }^{2}$ Das ist kein Vorwurf, sondern eine den politischen Verhältnissen geschul-

2 Einige bundesdeutsche Sprachwissenschaftler haben sich diese Mühe der Kontaktsuche auf Vertrauensbasis gemacht. Als schönes Beispiel sei auch hier Manfred W. Hellmann genannt, der sich stets um faire 
dete Tatsache. Nicht einmal vor Westbesuchern und sogar vor Familienangehörigen und guten Freunden aus dem anderen Teil Deutschlands machte die Furcht der DDR-Bürger, die Stasi höre mit, gänzlich Halt. Wer wusste schon, was der Gesprächspartner richtig oder falsch verstanden hatte und ohne Schaden für seine DDR-Partner anderen weitererzählte, womöglich schon bei den Kontrollen anlässlich der Ausreise oder der nächsten Einreise.

\section{Wortschatzfragen}

\subsection{Sprachspaltung oder regimespezifischer Bezeichnungswandel}

Die DDR war ein Staat mit einem den neutralen Betrachter noch im Nachhinein überwältigenden Abkürzungswesen, im Einzelnen sicherlich erklärungsbedürftig, aber als Tendenz des Sprachgebrauchs eher eine Eigenheit aller modernen deutschen (und vieler anderer) Bürokratien. Müssen dieselben Bezeichnungen immer öfter wiederholt werden, so bilden sich Abkürzungssysteme heraus, gewünschte und verordnete, aber auch ungeplanter Wildwuchs. ${ }^{3}$ Viele Abkürzungen gehörten tatsächlich zum Lebensalltag des DDRBürgers und haben deshalb Einzug auch in deren Alltagssprache gefunden. Außer den Abkürzungen der bekannten Partei- und Organisationsnamen gehörten zum Kern dieses Bestandes - mit gewissen Gebrauchsunterschieden zwischen Stadt und Land, aber auch zwischen Alt und Jung - vor allem wohl die folgenden: $A B F$ (Arbeiter-und-Bauern-Fakultät), $A B V$ (Abschnittsbevollmächtigter der Deutschen Volkspolizei, umgangssprachlich oft: der ABVer), $A D N$ (Allgemeiner Deutscher Nachrichtendienst), $B G L$ (Betriebsgewerkschaftsleitung, dazu: der BGLer), $B H G$ (Bäuerliche Handelsgenossenschaft, mit Läden in Dörfern und Kleinstädten), $B K V$ (Betriebskollektivvertrag), $E O S$ (Erweiterte [also zwölfklassige] Oberschule), GPG (Gärtnerische Produktionsgenossenschaft), DEFA (Deutsche Film-AG), LPG (Landwirtschaftliche Produktionsgenossenschaft), MAS (MaschinenAusleih-Station), MTS (Maschinen- und Traktoren-Station), NAW (Nationales Aufbauwerk), NVA (Nationale Volksarmee), $P G H$ (Produktionsgenossenschaft des Handwerks), $R G W$ (Rat für gegenseitige Wirtschaftshilfe), $S M A$ (Sowjetische Militär-Administration), $S V K$ (Sozialversicherungskasse, dazu der wichtige SVK-Ausweis für alle Arztbesuche), $T G L$ (Technische Normen, Gütevorschriften und Lieferbedingungen, parallel zu den alten $D I N$-Normen, noch in der BRD), $V d g B$ (Vereinigung der gegenseitigen Bauernhilfe), $V E B$ (Volkseigener Betrieb), VEG (Volkseigenes Gut), VP ([Deutsche] Volkspolizei, dazu der Vopo), $Z K$ (Zentralkomitee der SED). Ob beliebt oder unbeliebt, Abkürzungen gehörten zur öffentlichen und privaten Alltagssprache. Sie sind nicht vergessen, aber inzwischen längst ersetzt oder ergänzt durch ein kaum ärmeres Angebot in der Bundesrepublik. An ganz wenige Besonderheiten sei erinnert: Aus der Abkürzung des Staatsnamens DDR wurde die Bezeichnung der eigenen Nylon-Entwicklung abgeleitet: „Dederon“. Der allgegenwärtige „Marxismus-Leninismus“ hieß umgangssprachlich und unbeanstandet $M L$. Die im lebenslangen pädagogischen System der DDR für viele obligatorischen ideologischen Kurse, gewöhnlich langweilig und dogmatisch, wurden zum gelegentlichen Ärger der SED Maleweibi abgekürzt (Marxistisch-Leninistische Weiterbildung). Wer noch fre-

Urteile über die Differenzen der deutschen Sprache in Ost und West bemüht hat (vgl. Hellmann 1988). Auch Heinrich Scholz hat schon gute Beobachtungen über die "Sprache des täglichen Umgangs" in der DDR beigesteuert (Scholz 1964, vgl. S. 92).

3 Auf die Bedeutung der Abkürzungen im Ost-West-Sprachvergleich hat schon Hugo Moser sachlich und umsichtig aufmerksam gemacht, s. Moser (1962, S. 16f.). 
cher war, nannte die jahrelang auf allen Gebieten der Produktion und Forschung geforderten Pionier- und Spitzenleistungen knapp Pispilei.

Der Gebrauch und die Ablösung eingeübter Abkürzungen, die ja nicht nur optisch auffälliger sind als unverstümmelte Wörter, ist ein schönes Beispiel für den Bezeichnungswechsel an Epochenübergängen. Im Jahr 1990 war der Wechsel besonders auffällig, weil der Austausch von Ostlexemen gegen Westlexeme und von Ost-Abkürzungen gegen WestAbkürzungen nur einen regionalen Teilbereich des Deutschen betraf und im Osten bei aller Freude über den politischen Wechsel eine Art sprachlichen Phantomschmerz erzeugte. Eine ganze Welt von amtlichen Bezeichnungen wurde im Osten mit ihren Abkürzungen praktisch am Stichtag der Wiedervereinigung historisiert und lebt seitdem nur in den Erinnerungen an die vierzig Jahre fort. Manche nannten auch das einen Sprachwandel.

\subsection{Spezifische Wortbildungen}

\subsubsection{DDR-Wortschatz oder gemeinsame Traditionen}

Ebenso wie an DDR-spezifischen Abkürzungen konnten sich Besucher aus der Bundesrepublik an Wortbildungen stören, die ihnen ärgerlich, lächerlich oder einfach typisch östlich vorkamen. Das Potential ähnlicher Bildungen im westlichen Staat dürfte allerdings kaum geringer sein, ist aber bis heute nie unter dem Aspekt der Sprachkritik gesammelt vorgeführt worden. Die Wortbildungsregeln beider Seiten sind im Wesentlichen identisch, bieten also für prinzipielle Kritik am Sprachgebrauch nur des Ostens kaum linguistisch begründbaren Anlass. Die Kritik zielt auch hier auf den Staat, die Ideologie der herrschenden Partei und die so oft wiederholten Versuche semantischer Manipulation. Solche Versuche sollen also keineswegs in Abrede gestellt werden, sind aber in der Regel nirgends dauerhaft erfolgreich. Wörter wie Schutzwall und Friedenskampf sind in der DDR deshalb nie in die private Alltagssprache der Mehrheit eingedrungen, weil ihr propagandistischer Impetus offen zu Tage lag.

Beim Versuch der eindeutigen Zuordnung bestimmter Wörter zum eigenständigen Sprachgebrauch der DDR ist allerdings Vorsicht geboten. Solange wir keine historisch ausreichend gefüllten Textkorpora haben und die Auskünfte der einschlägigen Wörterbücher sehr lückenhaft sind, bleiben wir auf mühsame Einzelfallsuchen angewiesen, um wortgeschichtliche Fragen wenigstens vorläufig beantworten zu können. Alle Teile des Deutschen Wörterbuchs von Jacob und Wilhelm Grimm ( $\left.{ }^{1} \mathrm{DWB}\right)$ für die Stichwörter von $\mathrm{H}$ bis $\mathrm{R}$ und auch wichtige Abschnitte der späteren Bände stammen noch aus dem 19. Jahrhundert und werden in der gewohnten Form nie erneuert werden. Unser Urteil über den älteren Sprachgebrauch, der ja häufig genug dem der DDR zugrunde liegt, bleibt deshalb vielfach unsicher. An typischen DDR-Gebrauch kann man sich heute aber noch erinnern. Schauen wir uns einmal die folgenden 23 Beispiele an: Aktivist, allseitig, Datsche, Eingabe, Errungenschaften, Fünfjahr[es]plan, Jugendweihe, Kampfgruppe, Kaufhalle, Kollektiv, Kollektivvertrag, Nationalpreis, Objekt, Pionierleistungen, Planwirtschaft, Plast[e], Raumpflegerin, „unsere Menschen“, Volksbildung, volkseigen, Volkskammer, Werktätige, Zentralkomitee. Die aufgezählten Wörter werden jedem ehemaligen DDR-Bürger sehr vertraut klingen und vielen Bundesbürgern ebenso. Wenn sie in einem Text gehäuft auftreten, darf man sicher sein, dass der Text aus der DDR stammt. Aber alle diese Wörter besitzen eine ältere Gebrauchsgeschichte aus der gemeinsamen deutschen Vergangenheit, 
oft auch aus der NS-Zeit. ${ }^{4}$ Ihr Gebrauch wurde in der offiziellen Sprache der DDR gelegentlich bewusst umgeprägt, oft aber auch ohne Bedenken fortgeführt. Auf die Nachweise sei hier in knapper Form eingegangen. Neueste Belege, die die Nachwirkung solcher „DDR-Wörter" bezeugen, sind in den großen Korpora leicht zu finden und werden hier nur in wenigen Fällen angeboten:

- Aktivist ist in ${ }^{2}$ DWB behandelt und dort seit 1907 belegt. Nur der für vorbildliche Produktionsleistungen ausgezeichnete Werktätige ist ein Kind der DDR, semantisch angeregt durch den russischen Gebrauch.

- Allseitig ist im ${ }^{2}$ DWB seit dem 17. Jahrhundert belegt.

- Datsche kennt ${ }^{2}$ DWB seit 1879 , und der dänisch-preußische Offizier Helmuth von Moltke erwähnt schon 1856 die Datsche der Großfürstin Marie (Moltke, Briefe. Berlin 1900. Tei1 2, S. 47).

- Eingabe ist in der Bedeutung „Antrag [an eine Behörde] “ laut ${ }^{2}$ DWB seit Mitte des 18. Jahrhunderts gut bezeugt.

- Errungenschaft/en/ war ein Lieblingspropagandawort der DDR, aber im Besitzrecht schon seit dem 16. Jahrhundert belegt und im Sinne ,politischer oder sozialer Erfolg, Fortschritt" laut ${ }^{2} \mathrm{DWB}$ seit 1845 durchgehend in Gebrauch.

- FünfjahrlesJplan, im ${ }^{2}$ DWB seit 1931 für die Sowjetunion belegt und noch vor der Übernahme des Terminus in die DDR-Planwirtschaft auch für Argentinien, Brasilien und Japan. Auch dieses „DDR-Wort“ hat die DDR-Zeit überlebt: Fünfjahresplan zum Sparen titelte die BERL. ZTG. am 25.6.2009, S. 11.

- Jugendweihe, laut WIKIPEDIA ist das Wort (angeregt durch Eduard Baltzer) zuerst 1852 bezeugt. Die Bewegung für Jugendweihen war in den Zwanzigerjahren recht aktiv, in der NS-Zeit weitgehend verboten, nach dem Zweiten Weltkrieg in West und Ost wieder aufgelebt. Dementsprechend verlief der Gebrauch des Terminus in der Öffentlichkeit. Im Jahr 1954 wurde die staatlich geförderte Jugendweihe durch das Politbüro der SED in der DDR offiziell eingeführt und deshalb von vielen für eine DDR-Neuerung gehalten. Der erste Beleg im DWDS stammt derzeit aus dem Jahr 1934.

- Kaufhalle, in der DDR allgemein für die Großverkaufsstellen der HO (Handelsorganisation) und der Konsumgenossenschaft (Konsum), vergleichbar den heutigen Supermärkten, aber für große Markt- und Einkaufshallen im DWDS schon seit 1906 reich belegt.

- Kollektiv, im DWDS seit 1919 belegt. Kurt Tucholsky tadelte 1928 brieflich die Vereinsmeierei des Kollektivs (DWDS). Der in der DDR ungemein beliebte Verweis auf das Kollektiv hat inzwischen Schule gemacht: „Das war ein Erfolg des Kollektivs“, stellt [die erfolgreiche Pariser Kriminalbeamtin] Dupif klar (BERL. ZTG. 9.7.2009, S. 3).

- Kollektivvertrag, das Wort ist im DWDS schon für 1856 als ,internationaler Vertrag zwischen mehreren Partnern" bezeugt, im arbeitsrechtlichen Sinn dort seit 1905: Der Kollektivvertrag muss den Einzelvertrag mehr und mehr ersetzen (BERL. TAGEBLATT 1.3.1905, S. 13). In diesem Gebrauch ganz geläufig auch in der Weimarer

$4 \quad$ In vielen Fällen klärt darüber das DWDS auf. Für die neueste Wortgebrauchsgeschichte bietet auch das digitale Recherchesystem COSMAS-II vorzügliche Suchmöglichkeiten. 
Republik und von 1933 bis 1938 ebenso in der NS-Zeit, seit 1934 allerdings durch die NS-Forderung nach der Einzelverantwortung des „Betriebsführers“ allmählich gegenstandslos. Nach 1945 in beiden deutschen Staaten in arbeitsrechtlicher Funktion wieder aufgenommen. In der BRD später durch den Terminus Tarifvertrag verdrängt (letzte BRD-Belege für Kollektivvertrag im DWDS derzeit für 1972). In der DDR bis 1990 als Kollektivvertrag bzw. Betriebskollektivvertrag weiter in Gebrauch. Besonders in Österreich und Südtirol gilt Kollektivvertrag für tarifrechtliche Regelungen nach Ausweis der Korpora (DWDS und COSMAS II) bis heute, offenbar etwas seltener auch in der Schweiz.

- Nationalpreis, ebenfalls keine Neuerung der DDR. Nationalpreise des NS-Staats wurden schon 1933 verordnet (DWDS). Die DDR nahm diese Tradition der staatlichen Auszeichnungen sehr intensiv auf.

- objekt, in der DDR geläufig für Bauwerke, häufig für größere industrielle Anlagen und zu schützende Einrichtungen (dazu Objektschutz), aber auch von militärischen Anlagen und Zielen. Doch schon Georg Simmel kannte wirtschaftliche Objekte (Philosophie des Geldes, Leipzig 1900 [DWDS]), und in der Weimarer Republik galt es, grosse Objekte industrieller Natur in Deutschland zu erwerben (BERL. TAGEBLATT 9.3.1920, S. 4 [DWDS]). Im Zweiten Weltkrieg stürzten sich japanische Flugzeuge angeblich freiwillig auf feindliche Objekte (DWDS), und am 22.6.1940 meldet der Wehrmachtsbericht über Fliegerangriffe Sachschäden an nichtmilitärischen Objekten (DWDS). Am 31.12. desselben Jahres heißt es, militärische Objekte wurden nicht getroffen (DWDS).

- Pionierleistungen verlangte die DDR vor allem von Technikern und Wissenschaftlern, aber konstruktive Pionierleistungen der deutschen Autoindustrie wurden schon 1933 gerühmt (BERL. TAGEBLATT 5.2.1933, S. 21 [DWDS]).

- Planwirtschaft war ebenfalls keine Erfindung der DDR, das DWDS bezeugt Sache und Wort seit 1912 mit zahlreichen Belegen. Auch aus der Weimarer Republik liefern Studien über Planwirtschaft zahlreiche Belege und das setzt sich im NS-Staat fort: In den 1940 veröffentlichten Jahresberichten für die deutsche Geschichte (14. Jahrgang, S. 361 [DWDS]) wird ein Autor sanft getadelt, weil ihm ein volles Verständnis für die staatlich geleitete Planwirtschaft und die notwendige Kenntnis ihrer Leistungen fehlt und er die freie kapitalistische Wirtschaftsform überschätzt.

- Plast, Plaste, die Autobahnwerbung für Plaste und Elaste aus Schkopau hat der DDR reichlichen Spott der Besucher aus der Bundesrepublik eingetragen. In der BRD hatte man sich seit den 50er Jahren an die Bezeichnung Plastik (mit englischen und französischen Ursprüngen) gewöhnt. In der DDR gab es eine spürbare Tendenz, wissenschaftliche Fachwörter in die Allgemeinsprache zu überführen. Dafür sorgten die Wirtschaftsseiten der Zeitungen Hand in Hand mit Radio und Fernsehen. Plast und Plaste sind in Fachtexten mindestens seit dem letzten Kriegsjahr 1945 gebräuchlich. Dem schloss sich nach dem Krieg der Sprachgebrauch des Plaste-Booms zunächst in Ost und West an. Aber nur die DDR blieb den Plasten über die 50er Jahre hinaus treu (Schmidt 2000 b, S. 2031f.). 
- Raumpflegerin, laut WDG (4, 1974, S. 2959) „Neuprägung“ für „,Frau, die beruflich Räume saubermacht". Das DWDS findet das „neue“" Wort allerdings schon 1931 in Hans Reimanns „Vergnügliches Handbuch der deutschen Sprache" (Berlin 1931, S. 369); aber in späteren Auflagen hat Reimann es wieder gestrichen. 1964 wird ein Automat als transistorisierte Raumpflegerin erdacht (DIE ZEIT 13.3.1964, S. 43 [DWDS]). Schon 1985 wird resümiert: die Raumpflegerin als Bezeichnung für die Putzfrau war zunächst scherzhaft gedacht (DIE ZEIT 15.2.1985, S. 63), und noch 1993 stellt die „Gesellschaft für deutsche Sprache“ fest: Bemühungen seit 20 Jahren, diese Berufsgruppe mit Bezeichnungen wie „Raumpflegerin" [...] sprachlich aufzuwerten, könnten als gescheitert angesehen werden (TAZ 25.3.1993, 20 [COSMASII]); da bot die TAZ aber allein im Mai und Juni 1993 schon neun Belege für die neue Berufsbezeichnung aus einem Gerichtsprozess an. Leider haben offenbar die großen Korpora des IDS und des DWDS viel zu selten die Stelleninserate der Zeitungen berücksichtigt. Heinz Küpper schreibt nämlich über Raumpflegerin schon 1966 mit Beleghinweisen ab 1959: Um 1955 aufgekommen, als der zunehmende Mangel an Putzfrauen nach einem anfangs scherzhaft, später ernsthaft gemeinten Neuwort gesellschaftlicher Mehrgeltung suchen ließ. Heute in ganz Deutschland und Österreich geläufig, auch in Stellenangeboten (Küpper 1966, S. 186). In der DDR war die aufwertende Bezeichnung Raumpflegerin statt Putzfrau in Stellenanzeigen in festem Gebrauch. Für Österreich ist Raumpflegerin mindestens ebenso gut bezeugt. Auch in Südtiroler Tageszeitungen begegnet die Raumpflegerin in privaten und gewerblichen Annoncen bis heute: Raumpflegerin in Jahresstelle [...] gesucht (DOLOMITEN 31.5.2001, S. 20), Raumpflegerin für unsere Büroräume (ca. $200 \mathrm{qm}$ ) gesucht (DOLOMITEN 1./2. 6. 2002, S. 23), Fleißige Raumpflegerin (auch Bügeln) für 3 - 4 Stunden in der Woche in Meran gesucht (DOLOMITEN-MARKT 13.6.2009, S. 4). Sie unterliegt dort heute aber, vor allem im gewerblichen Stellenmarkt, einer erneuten auffälligen Konkurrenz durch die ,altmodischen“ Benennungen Haushaltshilfe, Hausmädchen, Mitarbeiterin zum Saubermachen, Putzfrau, Reinigungsfrau, Reinigungskraft, Zimmermädchen, Zugeherin, Zugehfrau.

- Unsere Menschen, die (sozusagen vormundschaftliche) Formel unsere Menschen war ein festes Requisit der DDR-Propaganda: Wir befähigen so unsere Menschen, dialektisch zu denken (FORUM 8.5.1958, S. 9), aber ein Erbstück aus der NS-Zeit. Die Formel findet sich schon 1942 in einem Wehrmachtsbericht und noch am 30.1.1945 unter den letzten verlogenen Propagandafloskeln zum Jubiläum der Machtübernahme durch das NS-Regime: [Feinde, die] unseren Menschen [...] Leid zufügen (DWDS). Google bietet bis heute auffällig viele Belege (allerdings oft Mehrfachnachweise) für unsere Menschen.

- Volksbildung, keine Erfindung der DDR-Volksbildungsministerin Margot Honecker oder ihrer Vorgänger, sondern eine ehrwürdige Forderung des deutschen Pädagogen Adolph Diesterweg (1790-1866). Das Wort ist früh belegt in Diesterwegs „,Rheinischen Blättern" (NF, Band 1, Essen 1830, S. 126 [DWDS]).

- volkseigen, das DWDS bietet Belege für volkseigenes Ideenleben schon im Jahr 1910 und für volkseigene Märchenstoffe 1927. Der Germanist Fritz Stroh benutzte die Naziunterscheidung volkseigen/volksfremd (Zeitschrift für Mundartforschung 15, 1939, S. 134). Die DDR ergänzte die emphatische Bedeutung durch die Wendung ins 
Ökonomische mit den Konnotationen „Enteignung“ und „das gehört doch eigentlich allen", was spürbare Folgen hatte. Auch dieses Wort hat den Übergang in die moderne Gegenwartssprache geschafft: General Motors ist bald ein volkseigener Betrieb, formuliert die BERL. ZTG. (30.5.2009, S. 3).

- Volkskammer, die Bezeichnung des über 40 Jahre lang einflusslosen Parlaments der DDR stammt aus demokratischer Tradition. ${ }^{1} \mathrm{DWB}$ gibt in der 1932 erschienenen Lieferung als Bedeutungsangabe für Volkskammer an: ,unterhaus, parlament aus allgemeinen wahlen im gegensatz zu oberhaus, herrenhaus, senat" und zitiert als Zeugen den Historiker und Abgeordneten der Frankfurter Nationalversammlung von 1848 Friedrich Christoph Dahlmann mit einem Frühbeleg aus dessen „Geschichte der Französischen Revolution bis auf die Stiftung der Republik" (Leipzig 1845, S. 144 $\left.\left[{ }^{1} \mathrm{DWB}\right]\right)$ : die bildung von senaten den volkskammern gegenüber. Auch Abgeordnete der Frankfurter Nationalversammlung verstanden deshalb ihr gewähltes Parlament und alle anderen aus Wahlen hervorgegangenen Landtage - als dem Volk verantwortliche ,deutsche Volkskammern“ (Wigard 1848-49, Bd. 8, S. 6211) im Gegensatz zum ebenfalls in Frankfurt tagenden Bundestag, der Kammer der Abgesandten der Regierungen des Deutschen Bundes. Die Mütter und Väter der Bundesrepublik Deutschland haben ihr Parlament in der Bezeichnungstradition der Regierungen des Deutschen Bundes benannt, die Begründer der DDR das ihre in der Tradition der $1848 \mathrm{er}$.

- Werktätige, die Substantivierung des schon seit dem 16. Jahrhundert geläufigen und auch von Goethe gebrauchten Adjektivs werktätig wird im ${ }^{1} \mathrm{DWB}$ leider nur für die NS-Zeit und die DDR behandelt. Die Beleglage im DWDS lässt deutlich erkennen, dass die NS-Propaganda den schon aus den 20er Jahren gut bezeugten linken Wortgebrauch des Substantivs Werktätige[r] nach 1933 intensiv übernommen hat. Nach 1945 wird er vor allem in der Russischen Besatzungszone und der DDR fortgeführt, um die Zusammengehörigkeit aller Arbeiter, Angestellten, der „Intelligenz“ und der „,werktätigen“ Bauern zu betonen. Die frühesten bisher nachgewiesenen Belege lauten: Jeder Werktätige erhält von mun an gerechten Lohn (zitiert aus einem Aufruf von Volksbeauftragten 1918, in: Gemeindeblatt des Kirchspiels Sakro, Dezember 1930, S. 4). Mit gesicherter Datierung zuerst in der [Berliner] Vossischen Zeitung (7.3.1921, S. 3 [DWDS]): [Harry Graf Kessler] erhofft alles Heil von der internationalen Verständigung der Werktätigen aller Länder. Neutral wertende Wortverwendungen in der Bundesrepublik bleiben lange selten, werden aber seit 1990 spürbar häufiger: die Stunde der [täglichen] Heimkehr der Werktätigen [nach der Arbeit] (Wolfgang Koeppen 1951 [COSMAS-II]); Krankheiten [...] vorzubeugen [...] durch die Plazierung der Werktätigen in ein weniger umweltgefährdetes Milieu (1984 Therapiewoche Nr. 8 [COSMAS-II]); Diepgen plaudert mit Werktätigen (DER SPIEGEL 16.1.1989, S. 25); Daher muß sich das Auge nicht ständig auf Unterschiede zwischen hellen und dunkleren Zonen einstellen. Der Werktätige an seinem Schreibtisch ermüdet deutlich langsamer (FAZ 1995 [COSMAS-II]); Nahrungsmittelbeschaffung ist bei diesen Werktätigen kaum zeitraubend (DIE ZEIT 4.4.1997, S. 80 [COSMAS-II]). Neutral wertende Belege aus der Schweiz begegnen in den 90er Jahren schon deutlich häufiger als in der deutschen Presse. 
- Zentralkomitee, das Zentralkomitee der Sozialistischen Einheitspartei Deutschlands, kurz das $Z K$, war mächtiger als die Volkskammer; selbst das Politbüro, das eigentliche Machtzentrum in der DDR, musste gelegentlich auf die Meinungsbildungen im ZK Rücksicht nehmen. Das ZK wurde fast täglich in den Zeitungen der SED erwähnt. Im Sprachgebrauch der DDR-Bürger gab es nur ein $Z K$. Das konnte bei allen zu Irritationen führen, die ein $Z K$ für eine sehr undemokratische Institution mit schlechtem Ruf hielten. Erst nach 1989 begegneten sie häufiger auch den Zentralkomitees anderer Organisationen und - bei historischem Interesse - der Fülle von Zentralkomitees, die schon im 19. Jahrhundert Namen und Ansehen besaßen.

\subsubsection{Kurzer Exkurs: Lexikalische Konnotationen}

Ein über Jahrzehnte eingeübter Wort- und Sprachgebrauch ist mit angenehmen und unangenehmen Konnotationen von Wörtern, formelhaften Sätzen oder Syntagmen, Kommunikationsformen und sogar grammatischen Eigenheiten verbunden, auch mit manchen daraus abgeleiteten Empfindlichkeiten. Unabhängig von sprachlichen Heimatgefühlen oder durch Sprache ausgelösten Aversionen haben sich in Ost und West während der Jahre der Trennung feste kommunikative Einstellungen, Erwartungen und Reaktionen herausgebildet und tradiert. Es gehört Erfahrung und Sprachwissen dazu, auch die Erwartungen und Reaktionen seiner Kommunikationspartner ausreichend sicher vorherzusehen und zu berücksichtigen. Wer zur Reflexion über die eigene Sprache bereit ist, sollte auch ihre historische Dimension in Betracht ziehen, erst dann kann das gemeinsame Spracherbe als gemeinsames begriffen werden. Was der Sprecher (manchmal auch der Linguist) nicht kennt, erklärt er gar zu gern zum hässlichen Produkt der Nachbarn über dem Fluss oder hinter den sieben Bergen, so z. B. die Großvieheinheit (Schmidt 2007, S. 7f.), die Kaufhalle, den Nationalpreis, die Planwirtschaft, die Plaste, die Volkskammer und manches andere, nicht nur Wörter aus unseren Aufzählungen. Deutsche aus der DDR und aus der Bundesrepublik könnten manches Vorurteil über den eigenen Sprachgebrauch und den der anderen überwinden, wenn sie erkennen, dass viel mehr, als der Uninteressierte denkt, in der gemeinsamen Sprachvergangenheit begründet ist, leider recht oft auch in der vergangenen, aber lange nachwirkenden Prägung einer ganzen Generation durch das Bezeichnungssystem des NS-Staats. ${ }^{5}$

\subsubsection{Gemeinsamer Wortgebrauch in Verfassungstexten}

In den wichtigsten Dokumenten der beiden deutschen Staaten aus dem Jahr der Teilung 1949, dem Grundgesetz (GG) der Bundesrepublik Deutschland (das die Möglichkeit des späteren Beitritts von ,anderen Teilen Deutschlands" offen hielt und so im Jahr 1990 auch zum Dokument der Wiedervereinigung wurde) und der Verfassung der DDR $\left(\mathrm{V}^{6}\right)$, findet sich trotz der grundsätzlichen politischen Unvereinbarkeiten eine ansehnliche Zahl von Wortgleichungen und gemeinsamen Verfassungsformeln. Die meisten sind durch die beiderseitige Abhängigkeit vom Text der Weimarer Verfassung von 1919 zu erklären, andere aber sind gemeinsame sprachliche Reaktionen auf die NS-Vergangenheit und betreten politisches Neuland in Verfassungsfragen. Insgesamt stellen die verfassungssprachlichen Gemeinsamkeiten oder Ähnlichkeiten einen interessanten Beleg für einen damals

\footnotetext{
5 Mehr hierüber und auch über nur vermeintliche DDR-Wörter in Schmidt (2007).

6 Zitiert als „V“ mit Hinzufügung der Jahreszahl der verschiedenen Textstufen.
} 
bewahrten Zusammenhalt zwischen Ost und West dar. Mit den folgenden Hinweisen soll dieser Zusammenhang angedeutet werden. So treffen die Verfassungstexte in oft lesenswerten Artikeln vergleichbare Bestimmungen über den Umgang mit gewichtigen Sachverhalten wie: Arbeitslosenhilfe GG 120[1] / Arbeitslosigkeit V 1949, 16; Besatzungskosten GG 120[1] / V 1949, 112; Enteignung GG 74[14]/V 1949, 24; Gemeineigentum GG 15 / V 1949, 27; Gemeinwirtschaft GG 15 / V 1949, 27; Kriegsbeschädigte GG 74[10]/ Kriegsgeschädigte V 1949, 26; Landschaftspflege GG 75[3] / V 1949, 26; (Mißbrauch) wirtschaftlicher Machtstellung GG 74[16] / V 1949, 24; Militarismus GG 139 / V 1949, 135; Nationalsozialismus GG 132[2] / V 1949, 144; öffentlicher Dienst GG 33[5] / V 1949, 3; Rasse GG 3[3] / Rassenhaß V 1949, 6; bisherige Reichsautobahnen GG 90[1] / V 1949, 124; Selbstverwaltung GG 28[2]/V 1949, 27; Sittengesetz GG 2 / Sittlichkeit V 1949, 133; Sozialversicherung GG 74[12] / V 1949, 16; System gegenseitiger kollektiver Sicherheit GG 24[2] / System der kollektiven Sicherheit V 1968, 6[4]; verfassungsmäßige Ordnung GG 9[2] / Verfassungsmäßigkeit V 1949, 66; Vergesellschaftung GG15 / V 1949, 27; Verwaltungsgerichtsbarkeit, Bundesverwaltungsgericht GG 95[1] / Verwaltungsgerichtsbarkeit, Verwaltungsgerichte V 1949, 138; Volksbegehren GG 29/V 1949, 3; Volksentscheid GG 29 / V 1949, 56; Volksvertretung GG 10[2]/V 1949, 4; Wiedergutmachung GG 74[9] / Wiedergutmachungsleistungen V 1949, 112; das Recht zum Widerstand GG 20[4] / das Recht und die Pflicht zum Widerstand V 1949, 4; Würde des Menschen GG 1[1] / menschenwürdiges Dasein V 1949, 19. Darunter sind einige Termini, die in der DDR nie oder nur sehr eingeschränkte Entsprechungen in der Rechtswirklichkeit gefunden haben (vgl. die Stichwörter Verwaltungsgerichte, Widerstand und Wiedergutmachungsleistungen). Die ebenfalls sehr interessanten Formelgleichungen der beiden deutschen Verfassungstraditionen sind in Schmidt (2004) vorgestellt worden.

\subsubsection{Hochkomplexe Lexeme}

Die im Abschnitt $3 \mathrm{zu}$ besprechende Formelvariation findet ein interessantes Anwendungsgebiet schon in der Wortbildung. Neben dem aktuellen lexikalischen Spielmaterial sind es vor allem gängige Formeln, die durch Zusammenschreibung oder als BindestrichKomposita, überwiegend auch durch zusätzliche Substantivierung ausschweifender, aber modischer Wortbildungen die Aufmerksamkeit der Leser gewinnen sollen. Obwohl im Ganzen kein politisch markiertes Stilmittel, wurde es nach der Wende auch in den Bereich lexikalischer Anspielungen auf die neue Lage eingeführt. Diese Wortbildungstechnik ist zwar relativ alt, aber erst in den letzten Jahrzehnten in der Bundesrepublik zu einem besonders beliebten journalistischen Verfahren geworden. In der DDR wurde sie vor der Wende wenig praktiziert. Jetzt gehört sie als journalistische Praxis zu den auffälligen „gesamtdeutschen Errungenschaften" in Ost und West. Nur in Buchveröffentlichungen bleibt sie noch selten.

Einige Beispiele: ${ }^{7}$

(1) Direkt-Substantivierung von Formeln:

Diehabenjaallesverkommenlassendenk'ich die ScheißModrowStasiConnection (TAZ 4.9.1990)

Zu Grundsatzfragen und zur Typologie s. Zemb (1978, S. 790-794); Fleischer/Barz (1995, S. 213); Schmidt (2000a). 
Den 1300 Dresdner Fans [bleibt] das ,,Ohne-Mielke-schafft-ihr's-nie “im Hals stecken (BERL. ZTG. 5.8.1996)

Ostzonensuppenwürfelmachenkrebs [Bandname nach einer Überschrift in der ersten Nummer der Bildzeitung 1952] (TS 15.1.1998)

Wir-sind-das-Volk besorgte [der Kreditwürdigkeit der DDR bei BRD-Banken] den Rest (TS 27.10.1994)

(2) Formeln als Determinative von Komposita:

Den erstaunten Was-es-im-Westen-so-alles-gibt-Blick (TS 13.12.1999)

Jetzt-ziehen-wir-nach-Berlin-Geschichte (TS 17.11.1998)

Nach anfänglicher Test-the-West-Phase (MM 29.10.1998) [Test the West war 1989/90 eine weithin, zum Beispiel auf allen Berliner S-Bahn-Höfen, präsente Zigarettenwerbung]

Ihre [der PDS] alte ,Die-Wessis-machen-uns-platt“-Nummer (TS 27.2.1999)

Der großmäuligen Fit-für-den-Weltmarkt-Wende-Rhetorik (TS 5.3.1996)

Wessi-nimmt-Ossi-Mandate-weg-Vorurteil (TS 24.9.1997)

Kein Schöner-wohnen-Wessi (BERL. ZTG. 26.6.1998)

Einig-Vaterland-Taumel (JUNGE WELT 24.3.1990)

\section{Gemeinsame Sprachspiele: Die Formelvariation}

\section{$3.1 \quad$ Zur Einführung}

Seit dem frühen 19. Jahrhundert wurde die parodierende Variation von Klassikerzitaten immer beliebter. ${ }^{8}$ Besonders bevorzugte Gegenstände der Variation wurden z. B. Schillers Lied von der Glocke (Fest gemauert in der Erden steht die Form, aus Lehm gebrannt), Goethes Faust und Goethes Lyrik (Über allen Gipfeln ist Ruh'). Auf diesem Hintergrund entwickelte sich das journalistische Zitierspiel, überwiegend in Zeitungsüberschriften, aber auch in Zeitungstexten, im Rundfunk und Fernsehen und seit einiger Zeit auch als Formuliertechnik von Buchautoren. Es scheint seit Jahrzehnten ein geradezu modischer Zeitvertreib von Redakteuren und Moderatoren zu sein, immer wieder Musterformeln zu entdecken und neue Möglichkeiten ihrer Variation zu ersinnen. Dabei stellen sie interessierte Leser vor die Frage, ob sie das verfremdete Zitat als Zitat erkennen und richtig zuordnen können. Beliebteste Variationsformeln sind die in den Medien besprochenen und deshalb bekannten Titel von Filmen, Büchern, Werbesprüchen, Wahlkampflosungen und anderen Politikerzitaten. Zum aktuellen Kernbestand der Musterformeln gehören die folgenden sechzehn Beispiele von deutschen Originalen bzw. Eindeutschungen fremdsprachiger Originale:

- Auf der Suche nach der verlorenen Zeit (Marcel Proust)

- Der Besuch der alten Dame (Friedrich Dürrenmatt)

- Endstation Sehnsucht (Tennessee Williams)

- Die Geburt der Tragödie aus dem Geiste der Musik (Friedrich Nietzsche)

- Gruppenbild mit Dame (Heinrich Böll)

- Ich denke, also bin ich (René Descartes)

8 Sehr anregend sind Wilss (1989), Burger/Buhofer/Sialm (1982) und Fleischer (1982). 
- Dein Mann, das unbekannte Wesen (Film 1970, Oswald Kolle)

- Der alte Mann und das Meer (Ernest Hemingway)

- München leuchtet (Thomas Mann)

- Die Plebejer proben den Aufstand (Günter Grass)

- Die Revolution [...] frisst ihre Kinder (Georg Büchner)

- Das Schweigen der Lämmer (Film, USA 1991, mit Anthony Hopkins)

- Schwerter zu Pflugscharen (Altes Testament)

- Stasi in die Produktion (Großkundgebung Berlin 4.11.1989)

- Die Wüste lebt (Dokumentarfilm von James Algar, USA 1953)

- Zu Risiken und Nebenwirkungen lesen Sie die Packungsbeilage und fragen Sie Ihren Arzt oder Apotheker (Pflichthinweis in TV-Spots zur Medikamentenwerbung)

Dazu finden sich dann vielfache Variationen wie:

- Auf der Suche nach dem veränderten Gen im Rapsfeld (TS 20.5.2000)

- Der Fluch der alten Dame (DER SPIEGEL 22.5.2006)

- Endstation Hauswand (TS 15.2.1997)

- Die Geburt der Karikatur aus dem Geist des Barock (DIE ZEIT 4.1.1985)

- Gruppenbild mit Männern (TAZ Osterausgabe 2006)

- Ich shoppe, also bin ich (WELT am SONNTAG 30.4.2006)

- Der Ostwähler - das unbekannte Wesen (DER SPIEGEL 15.4.2002)

- Der alte Mensch und der Hausarzt (DOLOMITEN 26. 5. 2005)

- München feuchtet (ZDF 8.12.2004) oder: Essen leuchtet (ARD 11.12.2005)

- Die Sozialfabrik probt die deutsche Zukunft (DIE ZEIT 23.10.2003)

- Die Korruption ernährt ihre Kinder (ZDF 8.12.2004)

- Das Schweigen der Schüler (TS 2.7.2002)

- Bomben zu Kunstwerken (TS 2.7.2002)

- Stasi in die Politik (TS 28.12.1998) und: Lobbyisten in die Produktion (Peer Steinbrück in: FAZ 12.1.2006)

- Die Wüste bebt (DER SPIEGEL 21.11.2005)

- Angesichts der bekannten Nebenwirkungen essen Sie die Packungsbeilage und sagen Sie nichts ihrem Arzt oder Apotheker (DER SPIEGEL 11.10.1999)

Je stärker die Variation das Basiszitat verfremdet, desto häufiger wird das Zitierspiel zum Rätselraten über die Variationsbasis. Dabei ist es durchaus möglich, dass der Autor der Variation nur von Kennern verstanden werden möchte, dann also in der Öffentlichkeit ein Spiel mit einer kundigen Elite unter Ausschluss der Öffentlichkeit treibt. Das Zitierspiel braucht immer neues Spielmaterial, sonst wird es langweilig. Bei der Suche nach neuen Texten spielen in der DDR tradierte oder geschaffene Texte eine erstaunlich produktive Rolle. Das betrifft nicht nur die in der DDR systematisch gepflegten Traditionen der kommunistischen Bewegung und auch einige sprachliche Übernahmen aus der Sowjetunion, sondern ebenso die Literatur und Dichtung von angesehenen und heute in ganz Deutsch-

Meist zitiert als: Der Mann, das unbekannte Wesen. 
land bekannten Autoren aus der DDR. Anspielungen auf die ungelösten Probleme der Wiedervereinigung begegnen in vielen der vorgestellten Variationsreihen auffällig oft. Die Techniken der Formelvariation sind vielfältig. Sie werden hier an einer Auswahl interessanter Beispiele vorgestellt. Viele der Basisformeln sind inzwischen in das gesamtdeutsche Sprachgedächtnis aufgenommen worden, auch die Variationstechniken sind in Ost und West längst verallgemeinert. ${ }^{10}$ Die Zahl der Beispiele muss hier eng beschränkt werden. Die Untergliederungen berücksichtigen deshalb nur die wichtigsten technischen Varianten.

\subsection{Formeln aus alter kommunistischer und sozialistischer Tradition}

Wir beschränken uns hier auf je ein Zitat aus zwei Grundtexten, dem Kommunistischen Manifest von Karl Marx und Friedrich Engels und der deutschen Fassung der „Internationale", der Hymne der (ehemaligen) kommunistischen Weltbewegung und über lange Jahre auch der wichtigsten Hymne der Sozialisten. Beide Texte waren natürlich immer auch in Westdeutschland bekannt, aber nur in Ostdeutschland gehörten sie zu den Erinnerungstexten jeder Schulbildung. Textteile daraus zählen noch heute zum stabilen Gedächtnispotential der meisten Deutschen, die dort aufgewachsen sind und lange genug die vom staatlichen Kindergarten bis zum Altersheim vorgesehenen Schulungen und Feierstunden der pädagogischen Provinz, die sich DDR nannte, genossen haben. Ein Unterschied zwischen West und Ost ist für unser Thema wichtig: Sprachspielerisch variiert wurden Zitate aus den rituellen Grundtexten der DDR dort in der Öffentlichkeit ebenso wenig wie Aussagen ihrer ,führenden Persönlichkeiten“. Spielerische Variation im Kernbereich der offiziellen Sprache der DDR konnte es nur im Schatten des politischen Lebens geben, allenfalls in aufregend benannten, aber genehmigten Kabarettprogrammen. Öffentliche Sprachspiele über wichtige DDR-Formeln gehören für die ehemaligen DDR-Bürger deshalb zu den ersten Früchten der neuen sprachlichen Gemeinsamkeit, zu den „Errungenschaften" der Nachwendezeit. Und erst jetzt zeigte sich, dass der ideologische Gehalt solcher Texte und Parolen in der Regel keinerlei Hindernis für ihre sprachspielerische Umformung bedeutet. Allein die Bekanntheit des Originals und der Witz seiner Umformung entscheiden über den Effekt des Spiels. Das leichte Gruseln, das manche Leser immer noch oder heute schon wieder bei der Lektïre solcher Texte und Zitate empfinden mögen, erhöht nur den Reiz und die Wirksamkeit der Textvariation.

\section{Ein Gespenst geht um in Europa - das Gespenst des Kommunismus}

Der erste Satz ${ }^{11}$ des Kommunistischen Manifests von Karl Marx und Friedrich Engels. London 1848 (Kuczynski 1995, S. 3).

Variationsbeispiele:

(1) Variation des Zitat-Endes (bei Beibehaltung des Zitatanfangs):

Ein Gespenst geht um in Europa, das Gespenst der Amerikanisierung (Herbstprospekt des Beck-Verlages, 2000)

Wieder einmal geht ein Gespenst um in Europa [...], das Gespenst der Gleichgültigkeit (MM 15.6.1999)

10 Ausführlichere Erklärungen und Beispiele in Schmidt (1997), Schmidt (2000c), Schmidt (2001a).

11 Die noch weit häufigere Variation des Schlusssatzes und Titelblattmottos „Proletarier aller Länder, vereinigt Euch!" wurde in Schmidt (2000c, S. 108-111) dargestellt. 
Ein Gespenst geht um in Europa - das Gespenst des Optimismus (DIE ZEIT 7.1.1999)

Ein Gespenst geht um in Europa, das Gespenst des Populismus (FAZ 24.7.2002)

Ein Gespenst geht um in Europa, das Gespenst des organisierten Verbrechens (FAZ 20.11.1998)

Ein Gespenst geht um in Europa: die Angst vor dem Aussterben (TAZ, Osterausgabe 2006)

Ein Gespenst geht um in Europa, [...] Karl Marx, gestorben 1863, lebt (SONNTAG AKTUELL 2.11.2008)

(2) Variation des Zitat-Endes und der Ortsangabe:

Ein Gespenst geht um im Adenauerhaus, aber diesmal ist es das von Peter Hintze (DER SPIEGEL 18.5.1998)

Ein Gespenst geht um in Deutschland - das Gespenst der Bajuwarisierung (TS 23.2.2002)

Ein Gespenst geht um in Deutschland: „Ich bin das Sparpaket" (BERL. ZTG. 17.4.1996)

Ein Gespenst geht um im geeinten Deutschland: „Ost-Identität" (DIE ZEIT 12.4.1996)

Ein Gespenst geht um in den Funkhäusern Deutschlands - das Gespenst des Absentismus (FAZ-Magazin 18.12.1998)

Ein Gespenst geht um in der Popmusik, und dieses Gespenst heißt Frau mit Gitarre (TAZ 16.12.1997)

Ein Gespenst geht um in Westberlin, es heißt ,, Verostung “ (DIE ZEIT 29.3.1996)

(3) Seltener ist die Variation des Zitat-Anfangs (bei meist freier Fassung weiterer Positionen):

Eine Angst geht um in Florida und es ist die Angst vor der Nichtberücksichtigung [der Wählerstimmen] (TS 22.11.2000)

Ein Killer geht um in Europa [...] Dr. Hannibal Lecter [In Ridley Scotts Film „Hannibal", 2000] (BERL. ZTG. 15.2.2001)

Ein Konzept geht um in Europa. Allerorten finden die sechs Bände „Lieux de memoire" des Historikers Pierre Nora Nachahmer (TS 14.5.2001)

Ein Phänomen geht um in Europa: Die Aufarbeitung des Kommunismus (DIE ZEIT 3.10.1997)

Eine Utopie geht um am Prenzlauer Berg in Berlin. Die Szene will einen elektronischen Kiez erbauen, das PrenzlNet (DIE ZEIT 20.9.1996)

(4) In syntaktisch freierer Fassung:

Anfang der neunziger Jahre ging ein Gespenst um in Europa: das Gespenst eines aggressiven Nationalismus (TS 13.3.1996)

Vor genau 150 Jahren ging ein Gespenst durch Europa, das „Kommunistische Manifest" der Revoluzzer-Dioskuren Marx/Engels (DER SPIEGEL 16.3.1998)

Ein Gespenst geht um. Es heißt Globalisierung (BERL. ZTG. 28.1.1998) 
Nicht mur in den Kolumnen über Vertreter der feinen Gesellschaft geht ein Gespenst um: Nachrichten über Erlauchte und Durchlauchte Adelige und deren sogenannte Häuser (BERL. ZTG. 22.10.1997)

Ein Gespenst geht um: Der Weltwirtschaft drohe eine Deflation (FAZ 3.11.1998)

An den Finanzmärkten der Welt geht ein Gespenst um: die Zinswende (DIE ZEIT 10.5.1996)

Vor 150 Jahren wurde das „Kommunistische Manifest" veröffentlicht. Und das Gespenst lebt weiter. Selbst in Amerika (TS 24.2.1998)

\section{Völker hört die Signale!}

Völker hört die Signale! / Auf zum letzten Gefecht! / Die Internationale / Erkämpft das Menschenrecht. Das ist der Refrain der „Internationale“ (Wacht auf, Verdammte dieser Erde!), des Hymnus der durch Karl Marx angeregten Internationalen Arbeiterassociation (1864 bis 1876). 1871 in französischer Sprache gedichtet durch Eugène Pottier, komponiert 1888, verdeutscht 1910 durch Emil Luckhardt.

(1) Aufforderungssätze mit Variation der Anrede (so die Masse der Varianten):

Aktionäre, hört die Signale (DIE ZEIT 27.1.2000)

Basketballer, hört die Signale! (TS 26.1.2000)

Familien, hört die Signale (MM 19.4.2002)

Funker, hört die Signale (DIE ZEIT 22.8.2002)

Genossen, hört die Signale! (WELT am SONNTAG 21.3.2004)

Händler, höret die Signale (DIE ZEIT 8.8.2002)

Käufer, hört die Signale! (DER SPIEGEL 25.10.1999)

Künstler, hört die Signale! (FAZ 19.7.2002)

Preußen, hört die Signale (WELT am SONNTAG 14.9.2003)

Startups, hört die Signale (BERL. ZTG. 30.11.2000)

Wessis, hört die Signale (TS 24.9.1999)

(2) Aufforderungssätze mit Auslassung der Anrede:

Hört die Signale (WELTBILD 06/2000 [Werbung für Lärmschutz])

Hört die Funksignale (FAZ 31.1.2001 [Über getarnte Propagandasender])

(3) Aussagesätze mit variabler Subjektstelle:

Alt-Sozialisten und Austernesser hörten die Signale (BERL. ZTG. 23.10.2001)

Das Volk hörte die Signale (MM 2.12.2003)

Volker [der Gründer des Berliner Grips-Theaters Volker Ludwig] hört die Signale (BERL. ZTG. 13.6.2007)

(4) Aufforderungssätze mit Variation der Objektstelle:

Völker, hört die Liberalen (FAZ 7.5.2001)

Völker, hört die Zitate, auf zum nächsten Konzert (FAZ 1.12.1999 [Musikkritik])

(5) Aufforderungssatz mit Variation aller drei semantisch wichtigen Wortstellen (aber mit Beibehaltung des Rhythmus und der Endreimwirkung):

Kanzler, füll die Regale (DIE ZEIT 7.6.2001) 


\subsection{Variationsformeln aus der DDR}

\subsubsection{Politische Formeln und Produktionslosungen der SED ${ }^{12}$}

Das politische Losungs- und Zitatenmaterial aus der DDR ist sehr umfangreich. Hier können in konzentrierter Form nur wenige Beispiele für die variierende Wiederaufnahme von Losungen des Ostens nach der Wiedervereinigung angeführt werden.

\section{Junkerland in Bauernhand}

Die Losung der Bodenreform in der Sowjetischen Besatzungszone seit Herbst 1945 erfährt bis in die Gegenwart neue Variationen:

Bauernland in Golferhand (BERL. ZTG. 19.2.2004)

Datschenland in Ossihand (BERL. ZTG. 15./16.6.1996)

Junkerland in Russenhand (BERL. ZTG. 14./15.6.2008)

Kirchenland in Bürgerhand (DIE ZEIT 8.2.2001)

Spekulantenland in Bauernhand (Forderung uckermärkischer Biobauern im Jahr 2008 [Google])

Spekulantenland in Junkerhand (DER SPIEGEL 3.1.2000)

\section{Die Partei, die Partei, die hat immer Recht!}

1950 dichtete Louis Fürnberg das alle SED-Mitglieder vom Selbstdenken befreiende „Lied der Partei": Sie hat uns alles gegeben / Sonne und Wind, und sie geizte nie. Fürnberg benutzte die Differenz von Recht und recht als Wortspiel. Der Refrain beginnt: Die Partei, die Partei, die hat immer recht!/ Und, Genossen, es bleibe dabei;/ Denn wer kämpft für das Recht, /Der hat immer recht. Aber Recht haben nun andere:

O ja. Die Partei, die immer recht hatte, macht einen selbstkritischen Eindruck (WOCHENPOST 11.4.1996)

Einheitspartei, die immer Recht hatte (DIE KIRCHE 26.6.1994)

Die Partei hat nicht mehr Recht (TS 23.1.2001)

Die Fabrik, die Fabrik, die hat immer recht (FAZ 8.10.2002)

Die FDP hat immer Recht (TS 15.5.1997)

Die Schalmei hat immer recht (TS 29.11.1996)

\section{Überholen, ohne einzuholen}

Eine Losung Walter Ulbrichts aus dem Jahr 1963 im erfolglosen Wettbewerb mit der Bundesrepublik. Die absurd klingende Formel wird noch heute wohl gerade deswegen in unterschiedlichen Zusammenhängen im Originalwortlaut gern zitiert, aber selten abgewandelt:

Abholen ohne einzuholen (BERL. ZTG. 20.2.2001 [Opernkritik])

Aufholen, ohne einzuholen (TS 12.4.2000 [über Tarifabschlüsse])

Ost-Berlin wird West-Berlin energiepolitisch bald überholen ohne aufzuholen (TAZ 30.11.1991)

12 Weitere politische Variationsformeln aus der DDR in Schmidt (1997) und Schmidt (2000c), in der zuletzt genannten Arbeit (S. 117-121) auch Variationen zur wichtigsten (biblischen) Oppositionslosung „Schwerter zu Pflugscharen“. 


\section{Meine Hand für mein Produkt}

Ein Beispiel für die zahlreichen, im Gedächtnis haftenden Produktionslosungen der DDR: Meine Hand für dein Produkt (BERL. ZTG. 17.8.2004)

Mein Gesicht für dein Produkt (BERL. ZTG. 24./25.1.2009)

\subsubsection{Variationsformeln aus literarischen Werken von DDR-Autoren}

Wir beschränken uns hier auf drei Beispiele. Für das erste, eines der produktivsten deutschen literarischen Variationsmuster überhaupt, wird hier nur eine Auswahl neuerer Belege nachgetragen. Das ältere Material ist dargestellt in Schmidt (2000c, S. 114-117).

\section{Kein Ort. Nirgends.}

Erzählung von Christa Wolf, 1979. Der Titel spielt auf eigenwillige Weise mit dem Begriff der „Utopia“, der eher optimistischen Verortung alter Phantasien über die ideale Gesellschaftsordnung in dieser Welt. Für Karoline von Günderode und Heinrich von Kleist, die Akteure der Erzählung, gibt es indessen keinen Ort der Erfüllung ihrer Hoffnungen. In den Variationen des Christa-Wolf-Titels äußern sich Bedauern und Dankbarkeit über das Fehlen von Lokalitäten oder über die Abwesenheit von Personen, Gefühlen, Zuständen. Auch die Verneinungsformen variieren.

(1) Orte und Gegenstände in der Substantivposition:

Kein Bild. Nirgends (BERL. ZTG. 6.3.2001)

Keine DDR. Nirgends (BERL. ZTG. 22.3.1999)

Kein Endlager, nirgends ( TS 18.12.2002)

Keine Handtasche, nirgends. Kein Hut, niemals. Hosenanzüge, meistens (SONNTAG AKTUELL 19.11.2006)

Kein Park. Nirgends (BERL. ZTG. 19.11.2001)

Kein [Brandenburger] Tor; nirgends (BERL. ZTG. 21.4.2002)

(2) Abstrakta, Äußerungen, Zustände:

Keine Allgemeinbildung. Nirgends (TS 24.10.2001)

Kein Ausweg. Nirgends (BERL. ZTG. 19./20.1.2002)

Keine Hoffnung, nirgends (TS 15.4.2002)

Kein Krieg. Nirgendwo (SONNTAG AKTUELL 16.9.2001)

Kein Rassismus. Nirgends (LITERATUREN Heft 6, 2002)

Keine These nirgends (FAZ 11.10.2003)

Kein Trost, nirgends (MM 17.10.2001)

Kein frischer Wind. Nirgends (DIE WELT 22.5.2008)

Kein Wort, niemals (TS 13.11.2001)

(3) Personen:

Kein Arbeiter. Nirgends (BERL. ZTG: 21.4.2004)

Kein Geißler, nirgends (TS 7.2.2001)

Kein Gott, nirgends (TS 12.1.2002)

Kein Gysi, nirgendwo (DER SPIEGEL 19.8.2002) 
Kein Held, nirgends (MM 10.9.2005)

Und kein Jammer-Ossi, nirgends (DER SPIEGEL 19.3.2001)

Kein Mensch nirgends (SONNTAG AKTUELL 20.6.2004)

KEIN NACHBAR NIRGENDS (TS 10.5.2002 [Bildunterschrift])

\section{Die neuen Leiden des jungen $W$.}

Das „Stück“ bzw. die Erzählung von Ulrich Plenzdorf war in der DDR eines der wichtigsten literarischen Ereignisse des Jahres 1973.

(1) Variiert wird fast ausschließlich die Position des Personennamens, meist unter Verzicht auf das erste Adjektivattribut:

Die Leiden des jungen B. [des US-Präsidenten Bush jun.] (FAZ 5.7.2003)

Die Leiden des jungen D. [des Fußballers Sebastian Deisler] (FAZ 28.4.2002)

Die Leiden des jungen Jan van Eyden [eines Radfahrers] (MM 31.8.2001)

Die Leiden des jungen Luftgitarristen (MM 9.5.2006)

Die Leiden des nicht mehr ganz jungen [Fußballers] Lothar Matthäus (MM 17.6.2000)

Das neue Leiden des alten P. [des deutschen Fußball-Pokals] (TS 6.5.2000)

Die Leiden der jungen $W$. [einer Filmfigur] (BERL. ZTG. 16.8.2004)

Die Leiden des jungen $Z$. [des Radfahrers Erik Zabel] (SONNTAG AKTUELL 8.7.2001)

(2) Bisher sehr selten mit Variation von Leiden:

Die alten Scheiben des jungen W. [die Schallplatten des Popstars Crischa Wagner] (SONNTAG AKTUELL 15.9.2002)

\section{Spur der Steine}

Roman von Erik Neutsch, 1964, Film und Filmverbot 1966. Erstaunlich ist die häufige Verwendung der Originalformel (hier in der ersten Gruppe), also ein Gebrauch, bei dem lexikalisch kaum etwas geändert wird, aber der Sachbezug neuartig ist (in der Regel wird in der Spitzenstellung der bestimmte Artikel hinzugefügt).

(1) Anwendung der Originalformel auf variierte Sachverhalte:

Spur der Steine: Vor zehn Jahren verschwand Honeckers Zitat [vom Pflaster auf dem Berliner Gendarmenmarkt] (TS 22.2.2000)

Die Spur der Steine [Joschka Fischer 1973 als Straßenkämpfer] (TS 4.1.2001)

Die Spur der Steine [Heinz Knobloch über Grabsteine] (TS 18.1.2001)

Spur der Steine [Markierung des Verlauf der Berliner Mauer] (TS 8.8.2001).

Die Originalformel syntaktisch eingebettet:

Im Internet die Spur der Steine [über gefilmte Architektur] (TS 15.3.1996)

Der Spur der Steine gefolgt [Steine des Berliner Schlosses] (TS 4.5.1998)

Die Spur der Steine an der Oder [Gedenksteine] (BERL. ZTG. 11./12.1998)

Auf der Spur der Steine [Trümmer des Potsdamer Stadtschlosses] (BERL. ZTG. 15./16.4.2000) 
Die Spur der Steine führt nach Henzendorf [Findlingspark] (TS 9. 1. 2001)

Ein Archäologe begibt sich [...] auf die Spur der Steine [mittelalterliche Bautrümmer] (BERL. ZTG. 24.7.2003)

Die Spur der Steine führt in die Rosenstraße [Berliner Denkmäler der Bildhauerin Ingeborg Hunzinger] (BERL. ZTG. 3.2.2005)

(2) Variation des Endglieds Steine. Hier werden nur solche Belege aufgeführt, deren Endglied zusätzlich durch den Reim auf Steine die Resonanzwirkung des Variationsmusters bezeugt. Da es bekannte ältere Filmtitel gibt (Die Spur des Falken mit Humphrey Bogart, Die Spur des Fremden mit Orson Welles), bleibt der Zusammenhang stärker abweichender Varianten (z. B. Spur der Blumen, Spur der Flaschen, Spur der Scherben, Spur der Stasi) mit unserem Muster unbeweisbar, wenngleich oft wahrscheinlich. Einschlägiges Material ist im Internet (Google) leicht zu finden. Hier also nur Beispiele für Varianten, deren Zusammenhang durch den Endreim auf Steine gesichert erscheint:

Die Spur der Beine [über Werbekalender] (SZ 21.11.2008)

Spur der Gesteine [Vorlesung am „Tag der Erde"] (BERL. ZTG. 25.4.2002)

Die Spur der Haine [über Olivenvermarktung] (BERL. ZTG. 27.6.2001)

Die Spur der Scheine [über Falschgeldumlauf] (DER SPIEGEL 10.3.1997)

Die Spur der Schweine [über Wildschweinplagen] (ARD 4.1.2004)

(3) Variation des Erstglieds:

Lektion der Steine [Kinderarbeit in einer Ziegelei] (DER SPIEGEL 7.8.2000)

Der Sinn der Steine [Zweck von Stonehenge] (TS 12.2.2003)

(4) Variierende Auflösung der Formelsyntax:

Staub der Steine, Spur des Feuers [Kunstwerke aus Staub] (MM 19.3.1997)

Steine ohne Spur [das Auswärtige Amt im Gebäude der Reichsbank und des SEDPolitbüros] (DIE ZEIT 20.1.2000)

\section{Deutschland und die Deutschen}

\subsection{Zur Einführung}

Die Gefahr einer Sprachspaltung zwischen Ost und West (eine im offiziellen Osten positiv, im Westen negativ beurteilte Entwicklung zu zwei eigenständigen Sprachvölkern) und die Möglichkeit, dass sich auf diese Weise im Osten eine für die innerdeutsche Kommunikation riskante Entwicklung zur Sprache des „Volks der Deutschen Demokratischen Republik" (so die SED-Formulierung in ihrer Verfassungspräambel von 1974) durchsetzen könnte, hat viele deutsche und auch ausländische Autoren lange beschäftigt. Es bleibt auffällig, dass zwar das Aufkommen und Wiederverschwinden des Bezeichnungssystems des NS-Staates untersucht wurde, dass aber die Frage einer durch solche Wechsel der Bezeichnungssysteme bedingten eingreifenden Änderung der gemeinsamen Sprache schon zu Beginn der NS-Zeit (zwischen Weimar und dem NS-Staat) und nach Überwindung der NS-Zeit (als Ausdruck der Sprachentnazifizierung) nur von sehr wenigen mit gleicher Intensität besprochen wurde und in der öffentlichen Diskussion kaum eine Rolle spielte. Die Annahme der Entstehung eines nach Einführung des NS-Bezeichnungssystems gegenüber der Weimarer Republik neuartigen Sprachvolks mit einer eigenständigen 
Sprache ist für die Jahre 1933 bis 1945 so kaum behauptet worden, obwohl zumindest die Emigranten sie sehr wohl für möglich halten konnten. Zwar hatte mitten in Deutschland zumindest Victor Klemperer mit großer Sorge die Tendenz zu einer eigenen Sprache des Dritten Reichs, der Lingua Tertii Imperii, beobachtet, aber nach dem Krieg hat sich das fachliche und öffentliche Interesse fast ganz auf die Untersuchung NS-typischer Wortschatzentwicklungen beschränkt. Die Idee, die Deutschen seien in den verhängnisvollen zwölf Jahren zu einem neuen Volk geworden (so wie das die Nazis ja propagierten) und danach, frisch entnazifiziert, gleich wieder zum ,völkischen“ und sprachlichen Zustand vor 1933 zurückgekehrt, wäre den meisten Zeitgenossen absurd vorgekommen. Dabei war der Abschied von der NS-Sprache für jeden spürbar. Im Mai 1945 wurde ja über Nacht

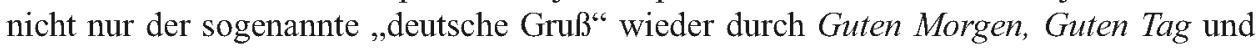
Guten Abend ersetzt, sondern es verschwand ebenso schnell ein ganzes Bezeichnungssystem von Naziwörtern, ganz radikal die zu NS und Reich gebildeten (mit der auffälligen Ausnahme der Deutschen Reichsbahn im Osten): von NSDAP (Nationalsozialistische Deutsche Arbeiterpartei) über $N S K K$ (Nationalsozialistisches Deutsches Kraftfahrkorps) bis NSV (Nationalsozialistische Volkswohlfahrt) und von $R A D$ (Reichsarbeitsdienst) über $R d I$ (Reichsverband der deutschen Industrie, nach 1945 abgelöst durch den $B D I$ ) bis $R S H A$ (Reichssicherheitshauptamt). Natürlich ebenso zahlreiche NS-Bezeichnungen ohne eingebürgerte Abkürzung aus der Verwaltungssprache, der Wehrmacht, der Wirtschaft, der Kulturverwaltung usw., darunter viele läppische, aber doch auch funktional wichtige, die hohe Gebrauchsfrequenzen besessen hatten. Niemand redete mehr über Reichsbräuteschulen, über den Reichsforschungsrat (Vorgänger des Wissenschaftsrats der Bundesrepublik) oder die ehemals wichtige Reichskleiderkarte (Zentner/Bedürftig 1993, S. 474, 476, 478). Im Jahr 1945 war der radikale Abgang des Naziwortschatzes und seiner üblichsten Abkürzungen ein Vorgang, der alle betraf. Aber die Bezeichnungswelt eines verbrecherischen Regimes ging unter, ohne dass die Fachwissenschaft oder das mit anderen Sorgen beschäftigte deutsche Volk dies ausdrücklich als Sprachwandel verstanden hätten. Nur den Brüdern und Schwestern im Osten, die ja unter sowjetischem Einfluss standen, trauten manche eine das ,gemeinsame Band der Sprache“ zerschneidende Entwicklung zu und wurden damit zum Opfer der SED-Propaganda.

Schon das den Linguisten vertraute Beispiel der Dialektforschung hätte ernste Zweifel an der These der Sprachspaltung nähren sollen. Dialektforscher haben bereits im 19. Jahrhundert erkannt, dass sich die Mundart von Dorf zu Dorf und von Person zu Person in der Aussprache und in bestimmten Teilen des Wortschatzes oder sogar der Grammatik unterscheiden kann, ohne die relative Einheit eines Dialekts ernstlich in Frage zu stellen. Nicht aus linguistischen Gründen, sondern aus politischer Sorge oder politischem Anspruch wurden Bezeichnungsänderungen in Ost und West, vor allem aber die der DDR, zum Problem. Heutige Deutsche im Alter von 70 oder 80 Jahren haben verschiedene politische Systeme mit vielen wichtigen systembedingten sprachlichen Besonderheiten durchlebt, doch sie müssen nicht im Ernst darüber nachdenken, ob sie mehrere tief einschneidende wirkliche Sprachwechsel (von der NS-Sprache zum Zonen- oder Bizonendeutsch und dann zu den gesonderten "Sprachen" der BRD und der DDR) erlitten haben. Nur ein immerwährendes Um- und Zulernen in Wortschatzfragen wurde ihnen in der Tat abverlangt, doch diese Notwendigkeit wird sie lebenslang, also auch in der Zukunft, begleiten. 
Das komplizierte Ost-West-Verhältnis erzeugte tatsächlich auch sprachliche Kuriositäten: Wer noch alte Exportartikel der DDR besitzt, kann darauf gelegentlich die Herkunftsbezeichnung Made in Germany entdecken (auf anderen dann auch das spezifischere Made in $G D R$ ). Auf Exportartikeln der Bundesrepublik erscheint aber (teilweise noch Jahre nach der Wiedervereinigung) weit häufiger die Formel Made in W[estern]-Germany. Der Oststaat, der eigentlich nur als Deutsche Demokratische Republik firmieren wollte, nannte sich aus wirtschaftlichen Gründen gern Germany, und der Weststaat, der das zeitweilige Ausweichen der offiziellen DDR auf die Bezeichnung Westdeutschland für eine Ausgeburt des Bösen hielt, konnte als Western Germany firmieren, wenn es um den Export, seine wirtschaftliche Existenzgrundlage, ging. Den sprachlich komplizierten Umgang mit dem Nachbarn zeigt auch eine Formulierung aus der Zeitung ,Die Welt“" (vom 16./17. 1993, S. 1), da heißt es: „Auftrag an die ostdeutsche Deutsche Waggonbau AG“. Ein Gegenbeleg für eine ,westdeutsche Deutsche Aktiengesellschaft“ liegt dem Autor dieses Artikels leider noch nicht vor.

Interessant ist aber auch die Wiederentdeckung des verbindenden „Deutschen“ durch die DDR, wenn die Chance nationaler und internationaler Wirkungen genutzt werden sollte, aber ebenso die Reaktion mancher bundesdeutschen Medien darauf. Als der DDR-Oberstleutnant Sigmund Jähn mit einer sowjetischen Rakete 1978 mehr oder weniger freiwillig in eine Erdumlaufbahn geschossen wurde und diesen Ausflug gesund überstanden hatte, lautete die Propagandaformel der DDR-Zeitungen: „Der erste Deutsche im All!". Die DDR-Bürger rieben sich überrascht die Augen, weil die Meldung deutlich an den seit 1974 durch die SED sorgfältig verdrängten Gedanken der Gemeinsamkeit der Deutschen erinnerte. Aber die Formulierung ging sofort auch in das bundesdeutsche Sprachgedächtnis ein. Man variierte sie in der Namensstelle statt in der Ordnungszahl und wandte sie noch Jahre danach auf den eigenen Mann an: „Ulf Merbold, der erste Deutsche im All!“ hieß es in der Sendung „Kult am Sonntag" am 6.1.2008 im ZDF in einer Rückschau auf die 80er Jahre.

Die Eigenschaft, „Deutscher“ zu sein, ist bekanntlich durch das Grundgesetz auch den Bürgern der DDR zugesichert worden, da auch sie im Gebiet des Deutschen Reichs nach dem Stand von 1937 lebten (Grundgesetz, Artikel 116, 1). Die Bundesrepublik ist der erste Staat der deutschen Geschichte, der „Deutschland“ in den offiziellen Staatsnamen aufgenommen hat, sozusagen als patentierte Eigenbezeichnung. „Deutschland“ war früher immer ein eher alltagssprachlicher Ausdruck für ein Land der Sehnsucht, eine Hoffnung der demokratischen 48er auf die kulturelle, sprachliche und (damals) eventuell auch politische Zusammengehörigkeit der Deutschsprechenden, wie sie nicht nur Hoffmann von Fallersleben bewegte, als er am 26.8.1841 auf Helgoland sein „Lied der Deutschen“ dichtete. Erst das Bestreben, nicht an die früheren Reichskonzepte und den Namen des „Deutschen Reichs" anzuknüpfen, hat 1949 die Staatsgründer in Ost und West bewogen, neue Namen für ihre vermeintlichen Provisorien zu finden. Der Rückgriff auf das alte Hoffnungswort lag nahe. Im Westen führte das zur Kombination des neuen Ländernamens mit dem Begriff für die Staatsform „Bundesrepublik“, im Osten erfand man die „Deutsche Demokratische Republik“, aber zunächst ohne dem Rückgriff auf „Deutschland“ ganz auszuweichen: Der erste Verfassungsentwurf für die DDR von 1946 (Schmidt 2004) hat neben dem Artikel 1: „Deutschland ist eine unteilbare demokratische Republik" noch andere Bestimmungen, die auf „Deutschland" referieren, so den schönen Artikel 9, der die 
Freizügigkeit der Deutschen garantiert: ,Jeder Bürger hat das Recht, sich an einem beliebigen Ort Deutschlands niederzulassen" und den ebenso bemerkenswerten Artikel 96: „Deutschland bildet ein einheitliches Zoll- und Handelsgebiet, umgeben von einer gemeinschaftlichen Zollgrenze". Diese Formulierungen wurden wörtlich auch in die erste Verfassung der DDR von 1949 übernommen. Sie bezeugen, wie leicht „Deutschland“ damals noch über die Lippen der SED-Funktionäre gehen konnte.

Doch die Zeiten änderten sich, nicht nur in der DDR, sondern auch in der Bundesrepublik. Die DDR setzte nach dem Mauerbau in der täglichen Praxis und natürlich in der Propaganda zunehmend auf konsequente Abgrenzung, und die Bundesbürger identifizierten ebenso zunehmend ihren Staat mit einem Deutschland, das - vom Westen her gedacht die DDR und ihre Bürger aus diesem Begriff ausschloss. Obwohl die Erfahrung und die Rede von der Spaltung Deutschlands alt ist und schon 1848 die Abgeordneten der Frankfurter Nationalversammlung ${ }^{13}$ bewegte, wird gelegentlich vergessen, dass die Spaltung Deutschlands zumindest zwei Teile hervorbringen musste, deren Bevölkerung man es hätte überlassen sollen, ob sie sich weiterhin als Teil Deutschlands verstehen wollte. Der östliche Staat wurde in der Bundesrepublik aber oft nicht mehr mitbedacht, wenn es um Deutschland oder gar „ganz Deutschland“ ging. Das entsprach zwar nicht dem Duktus der politischen Erklärungen und der Sonntagsreden, wurde aber für viele tägliche Praxis. Deshalb wurden Formulierungen möglich wie:

- Die Verfilmung, Klein Erna auf dem Jungfernstieg' (1969) [...] machte die norddeutsche Figur in ganz Deutschland bekannt (MM 14.4.2007)

- Durch den Aufbau der ersten neuen Nachkriegskasernen in Baumholder [...] hatte es einen Bauboom für Baubetriebe in ganz Deutschland gegeben (3sat 9.11.2007)

- In einem Stollen bei Oberried/Schauinsland liegen seit den 70er Jahren Kopien von Kulturgütern aus ganz Deutschland (SWR, Baden-Württemberg aktuell 6.3.2009)

Klein Erna war zwar auch in Ostdeutschland schon vor dem Zweiten Weltkrieg bekannt, aber nicht durch einen Film von 1969, der dort nicht gezeigt werden konnte. Am Bau der Kasernen in Baumholder waren bestimmt keine Baubetriebe aus der DDR beteiligt, und auch Kopien von Kulturgütern aus der DDR lagerten 1970/80 gewiss noch nicht im Barbarastollen von Oberried im Schwarzwald. Wer so formuliert, steht in einer bis heute recht stabilen alten BRD-Tradition, die ,ganz Deutschland" mit dem Stolz der Wirtschaftswunderer auf die Bundesrepublik reduzierte (Schmidt 2001b, S. 568).

\subsection{Zwei Formelvariationen zum Deutschlandthema}

Wie schnell nach der Wende gern auf die „Becherhymne“, die in früheren Zeiten in der Bundesrepublik gemiedene und in der DDR seit den 70er Jahren nicht mehr gesungene östliche „National“-Hymne, zurückgegriffen wurde, zeigt der Gebrauchswert ihrer ersten Zeilen als Buchtitel bedeutender bundesdeutscher Autoren: Auferstanden aus Ruinen (Dieter Zimmer, Stuttgart 1989) / Und der Zukunft zugewandt (Wolfgang Schäuble, Berlin 1994) / Lass uns dir zum Guten dienen (Willy Wimmer, ${ }^{14}$ Berlin 1994) / Deutschland, einig Vaterland (Hellmut Diwald, Berlin 1990). Als Hymnendichter zum Thema der Einheit Deutschlands war der längst verstorbene Autor, wenn auch noch nicht zu Lebzeiten,

13 So Robert Blum in Wigard (1848-49, Band 1, S. 36); vgl. Schmidt (2002, S. 61).

14 Der Autor war Staatssekretär im Bundesverteidigungsministerium. 
offenbar erfolgreicher als in seinem letzten Amt als Kulturminister der DDR, deshalb sei hier einmal die vierte Zeile seines umstrittenen Liedes als Variationsmuster genauer vorgestellt.

\section{Lass uns Dir zum Guten dienen, Deutschland, einig Vaterland}

Aus dieser vierten Zeile der ersten Strophe des Liedes wurde fast ausschließlich nur die zweite Hälfte variiert, und auch deren Variationsfrequenz bleibt weit hinter der der ersten Zeile Auferstanden aus Ruinen (Schmidt 1997, S. 42f.; 2007, S. 9) zurück. Aber es lohnt sich, auch die in den Variationen der vierten Zeile ausgedrückte Zeitkritik und Spottlust zur Kenntnis zu nehmen.

(1) Nur das Adjektivattribut ist ausgetauscht (sehr selten in Kombination mit Variation wie in Punkt 2):

Deutschland, kleinlich Vaterland (DIE ZEIT 1.10.2003)

Deutschland schwierig Vaterland (Buchtitel: Richard Schröder, Freiburg 1993)

Deutschland, teures Vaterland (TV: Hessischer Rundfunk 3.7.1997)

Deutschland uneinig Vaterland (TS 15.11.1996, S. 11; 14.5.1998)

Deutschland, ungleich Wirtschaftsland (DIE ZEIT 22.11.2001)

(2) In der Mehrzahl der Variationen wird das Endglied abgewandelt:

Deutschland, einig Christenland? (rtv [TV-Zs.] Nr. 43, 1998)

Deutschland, einig Einwanderungsland (DIE ZEIT 26.4.2001)

Deutschland, einig Fußball-Land (MM 2.7.1996; FAZ 16.6.2006)

Deutschland, einig Köterland (DIE ZEIT, Beilage Leben, 29.7.1999)

Deutschland, einig Memmenland (BERL. ZTG. 17.7.1998)

Deutschland, einig Mütterland (DER SPIEGEL 17.9.2007)

Deutschland-einig Narrenhaus (BERL. ZTG. 28.8.1996)

Deutschland, einig Schnäppchenland (RHEIN. MERKUR 15.8.1997)

Deutschland, einig Steuersünderland (DER SPIEGEL 25.9.1995)

Deutschland, einig Stillstandsland (DIE ZEIT 24.1.2002)

Deutschland, einig Währungsland (BERL. ZTG. 4.3.2008)

Deutschland, einig Zockerland (DIE ZEIT 25.11.1999)

Selten einmal das Anfangsglied:

Europa, einig Bildungsland (TS 27.8.1998)

(3) Relativ selten (am häufigsten in Überschriften) wird Deutschland ganz weg gelassen:

Einig Euroland (TS 2.1.2002)

Freude zwischen Rostock und Garmisch - einig Fußball-Land (TS 2.7.1996)

Einig Katerland (DIE ZEIT 2.10.2002)

Einig Radioland (TS 6.7.2000)

Einig Raserland (BERL. ZTG. 30.10.2007)

Einig Witzigland (BERL. ZTG. 15.10.1998) 
Ein einig Land von Beamten (TS 20.12.1995)

Kein einig Fernsehland (TV Spielfilm 1996, Nr. 4)

Kein einig Malerland (DIE ZEIT, ZEITmagazin 29.8.1997)

Kein einig Vaterland (Buchtitel: Günter Gaus, Berlin 1998)

\section{Wir sind das Volk!}

Diese wichtigste Losung der Demonstranten vom Herbst 1989 in Leipzig, Berlin und anderen Städten ist wohl zuerst 1848 im Verlauf der Pariser Februarrevolution formuliert worden (Wir sind das Volk, und das Volk ist Alles!, in dieser Formulierung im März 1848 kritisch zitiert durch „Briefe aus Paris“ in: „Die Grenzboten“, 1. Jg., 1. Semester. Leipzig 1848, S. 588 ${ }^{15}$ ). Ihre frühe Verbreitung verdankte die Losung aber dem Gedicht „Trotz alledem!" (Ferdinand Freiligrath, Neuere politische und soziale Gedichte. Köln 1849), das auch in der jungen DDR auf Feierstunden gern rezitiert worden war. Das Gedicht selbst trägt Freiligraths Entstehungsangabe „Düsseldorf, Anfang Juni 1848“. Im letzten Vers heißt es: Wir sind das Volk, die Menschheit wir. Ausgangspunkt des modernen Variationsspiels war die Aufnahme der ersten Satzhälfte von Freiligraths Formulierung. Der nächste wichtige Schritt war der Ersatz des bestimmten Artikels durch das betonte Zahlwort: Wir sind ein Volk. Damit nahm man wohl bewusst eine Formulierung von Friedrich Schiller auf: Wir sind ein Volk, und einig wollen wir handeln (Wilhelm Tell, Akt 2, Szene 2). Darauf folgte der Austausch von ein Volk durch Deutschland.

Hier können nur wenige Beispiele eines überaus reichen Variationsschatzes angeführt werden. Die Anordnung folgt den Wörtern wir, sind, Volk. Die Variation des bestimmten Artikels (ein, kein oder Nullform) ist in den anderen zugehörigen Reihen zu sehen. Mehrfach begegnen weitere Variationen in Beispielen einer Reihe. Extrem ausgebaut ist allerdings nur die dritte Gruppe mit der Variation von Volk. Zusätzliche Einflüsse anderer Muster (Der Staat bin ich, Ich bin ein Berliner, Das Kaninchen bin ich, We are New Yorker!) sind möglich, werden aber hier nicht ausgewiesen. Ähnlich wie auf der großen Berliner Demonstration vom 4. November 1989 zwischen Alexanderplatz und Palast der Republik wird die Revue der Losungen erlebbar als Folge von Dialogszenen:

(1) Variation von Wir (mit jeweils passender Verbform und gegebenenfalls weiteren Variationen):

Ich bin das Volk (F. X. Kroetz, Volkstümliche Szenen aus dem neuen Deutschland. In: Berliner Ensemble, Drucksache 13/14. Berlin 1995, S. 473-564)

Ich bin ein Volk (TS 10.3.1998)

Ich bin Volker (auch als Fahrschulwerbung im Internet 14.5.2009)

Ich bin Deutschland (DER SPIEGEL 24.4.2006)

Ich bin auch Deutschland (FAZ 26.1.2006)

Ich bin ein Euro (DIE ZEIT 10.9.1998)

Du bist Deutschland (DER SPIEGEL 2.1.2006)

Du bist Ludwig Erhard (DER SPIEGEL 2.1.2006)

Du bist Otto Lilienthal (DER SPIEGEL 9.1.2006)

15 Den Hinweis auf diesen Beleg und die französische Herkunft verdanke ich meiner Kollegin Gabriele Hoppe (Schwetzingen). 
Sie sind das Volk (DIE ZEIT 20.8.1998)

Wir sind kein Volk ([Buchtitel] Wolfgang Herles, München/Zürich 2004)

Ihr seid kein Volk (BERL. ZTG. 4.4.2000)

Hans Bauer ist das Volk (MM 12./13.4.1997)

(2) Variation der Tempus- und Modusform des Verbs:

Wir waren das Volk (BERL. ZTG. 5.10.1999)

Wir waren nicht ein Volk (BERL. ZTG. 7.1.1999)

[Sie dachten,] sie seien das Volk (DER SPIEGEL 25.9.1995)

Wir waren Deutschland (MM 15.7.2006)

(3) Variation von Volk:

(3a) Mit dem bestimmten Artikel vor der Variation (der Hauptgebrauch):

Wir sind die Bank (DER SPIEGEL 31.8.1998)

Wir sind das Eigentum (BERL. ZTG. 9.10.1996)

Wir sind das Geld (DIE ZEIT 31.5.1996)

Wir sind die Kirche (BERL. ZTG. 21.1.1995)

Wir sind die Kneipe (FAZ 24.10.1998)

Wir sind das Milieu (DIE ZEIT 15.12.1995)

Wir sind das Netz (TS 21.1.1998)

Wir sind der Sender (FAZ 4.1.2001)

Wir sind die Shell (WOCHENPOST 26.10.1995)

Wir sind der Staat (TS 24.8.1999)

Wir sind ein Wörterbuch! - Wir sind das Wörterbuch"! (Titel von Burkhard Schaeder in: Zeitschrift für germanistische Linguistik, 22, 1994, S. 58)

Wir sind das Wunder (WOCHENPOST 11.4.1996)

Wir sind die Zukunft (WOCHENPOST 12.12.1996)

(3b) Ohne das Artikelwort vor der Variation (vgl. die Gruppen 1 und 2):

Wir sind Bundeskanzler (SONNTAG AKTUELL 21.1.2006)

Wir sind Bundestrainer (MM 8.4.2006)

Wir sind Europa (MM 29.12.2006)

Wir sind Heidelberg (MM 10.4.2007)

Wir sind Hollywood (TV-Sender „Das Vierte“, 8.1.2006)

Wir sind Kaiser [Suppenreklame] (ORF1 17.6.2009)

Wir [die Angestellten] sind Karstadt (Demonstrationsplakat in: ZDF reporter 11.6.2009)

Wir sind Mannheim (MM 31.3.2006)

Wir sind Marmelade (BERL. ZTG. 20./21.10.2007)

Wir sind Medaillenspiegel (SONNTAG AKTUELL 26.2.2006)

Wir sind Nobelpreisträger (BERL. ZTG. 1.1.2007)

Wir sind Oscar [der „Oscar“ für „Das Leben der Anderen“] (ZDF Frontal 6.3.2007) 
Wir sind Papst (Zitat aus der Bildzeitung in: ARD 11.4.2007)

Wir sind Stoiber (BERL. ZTG. 22.2.2007)

\subsection{Schlussbemerkung}

Der historische Deutschland-Begriff war über Jahrhunderte staatspolitisch offen und zielte weniger auf eine staatliche Realität als auf die fragwürdige, sehr differenzierte Gemeinschaft der Deutschsprechenden und auf ein Gebilde der Hoffnung. Bis weit in das 20. Jahrhundert stieß die Frage der beiden Weimarer Dichter Deutschland, aber wo liegt es? deshalb für viele erneut auf die pessimistische Antwort Kein Ort. Nirgends. Der Traum vom anderen Deutschland, vom besseren Deutschland, vom ganzen Deutschland oder vom neuen Deutschland wurde oft geträumt, aber dieses Deutschland blieb irreal, wenn man nicht sehr bescheidene Wünsche hatte oder allzu unkritisch und selbstbezogen glaubte, das eigene sei das ganze. Real waren - für sehr lange Zeit - das Deutsche Reich und realsozialistisch für eine Generation die $D D R$. Der 1848 politisch umstrittene Begriff und Name Großdeutschland, ${ }^{16}$ aufgewärmt durch die NS-Propaganda seit 1921, wurde in der NS-Zeit 1938 für sieben Jahre zwar zur Staatsbezeichnung, bis heute eine philatelistische Kuriosität, in Wirklichkeit für viele Betroffene ein Schreckgespenst. Die Mütter und Väter des Grundgesetzes entschlossen sich, ihr Deutschland, das durchaus als der politische und kulturelle Erbe des Deutschen Reichs Weimarer Prägung gedacht war, so auch zu nennen. Sie folgten damit einer langen alltagssprachlichen Tradition: Der kritische Journalist Peter Bender hat kürzlich das Deutschlandbild und das Bild der Deutschen charakterisiert, in das er hineingeboren wurde: „Ich bin 1923 geboren, wuchs in einem Deutschland auf, das von Freiburg bis Tilsit reichte. Deutschland war keine Frage, sondern selbstverständlich als die staatliche Form, in der die Deutschen 1eben" ${ }^{6}{ }^{17}$ Solche Erfahrungen werden binnen Kurzem vergessen sein. In der DDR blieb das Neue Deutschland über 40 Jahre nicht nur ein Zeitungstitel, sondern ein Wunschgebilde, allerdings in mindestens zwei Versionen, denn die Vorstellungen der herrschenden Partei unterschieden sich völlig von denen der Bevölkerungsmehrheit. Lange Zeit war dort das Bild Deutschlands ein Gegenbild zur erlebten Wirklichkeit, nicht irreal, aber unerreichbar. Eine neue Realität gewann Deutschland für beide deutsche Staaten, als sich die Mauer und der Stacheldraht öffneten. Aber nun versagten vielen Deutschen plötzlich die Ideale vom gemeinsamen anderen, besseren und neuen Deutschland. Und die Schuld sahen sie nicht nur in der Politik, in der Wirtschaft oder beim deutschen Nachbarn, sondern auch in der Sprache, die man so lange kaum noch gemeinsam geübt hatte. So beginnen Deutsche also schon wieder, die eigenen Träume zu träumen, die Idylle der getrennten Vergangenheit zu pflegen, auch die der getrennten Kultur, Literatur, Sprache und Kunst, und wollen immer noch nicht in die Verantwortung für eine tatsächlich gemeinsame Geschichte, Gegenwart und Zukunft, für die gemeinsame Sprache, Kunst und Literatur eintreten. Die These Wir waren Deutschland ist noch nicht angenommen, die Losung Wir sind Deutschland bleibt eine Aufgabe, auch in Europa.

16 Seit dem 16. Jahrhundert belegt und in politischem Sinn schon für Jean Paul, s. ${ }^{1} D W B$. Vgl. auch Schmitz-Berning (2000, S. 287f). Der NS-Gebrauch eingehend dargestellt durch Eitz/Stötzel (2007, S. 277-294).

17 Peter Benda: Bundesrepublik oder Deutschland? Auch ein Problem sozialer Ungleichheit. In: FAZ 24. 9. 2008, S. 36. 


\section{Benutzte wissenschaftliche Literatur, Wörterbücher und Korpora}

Burger, Harald/Buhofer, Annelies/Sialm, Ambros (Hg.) (1982): Handbuch der Phraseologie. Berlin/New York.

COSMAS II: Textkorpora und Recherchesystem des Instituts für Deutsche Sprache in Mannheim (IDS). [Abrufbar unter: www.ids-mannheim.de].

Dieckmann, Walther (1969): Sprache in der Politik. Einführung in die Pragmatik und Semantik der politischen Sprache. Heidelberg. (Sprachwissenschaftliche Studienbücher. Zweite Abteilung).

'DWB (1854-1971): Deutsches Wörterbuch von Jacob Grimm und Wilhelm Grimm. Leipzig: S. Hirzel. XVI Teile und Quellenverzeichnis in 33 Bänden.

(Dazu:) [Einbandtitel: Der Digitale Grimm] Deutsches Wörterbuch. Elektronische Ausgabe der Erstbearbeitung. Frankfurt am Main: Zweitausendeins. 2 CD-ROMs, Begleitbuch, Benutzerhandbuch. [Auf dieser Ausgabe beruht die Internetfassung des DWB].

${ }^{2}$ DWB (1983ff.): Deutsches Wörterbuch von Jacob Grimm und Wilhelm Grimm. Neubearbeitung. Hg. von der Akademie der Wissenschaften der DDR in Zusammenarbeit mit der der Akademie der Wissenschaften zu Göttingen (ab Band 2: Hg. von der Berlin-Brandenburgischen Akademie der Wissenschaften und der Akademie der Wissenschaften zu Göttingen). Leipzig (später Stuttgart): S. Hirzel. 9 Bände [Band 4 und 5 sind in Arbeit].

DWDS: Digitales Wörterbuch der Deutschen Sprache. Textkorpora und Recherchesystem der Berlin-Brandenburgischen Akademie der Wissenschaften (BBAW). [Abrufbar unter: www.dwds.de].

Eitz, Thorsten/Stötzel, Georg (2007): Wörterbuch der „Vergangenheitsbewältigung“. Hildesheim/Zürich/ New York.

Fleischer, Wolfgang (1982): Phraseologie der deutschen Gegenwartssprache. Leipzig.

Fleischer, Wolfgang/Barz, Irmhild (1995): Wortbildung der deutschen Gegenwartssprache. 2. Auflage. Tübingen.

Hellmann, Manfred W. (1976): Bibliographie zum öffentlichen Sprachgebrauch in der Bundesrepublik Deutschland und der DDR. Düsseldorf. (Schriften des Instituts für deutsche Sprache 16).

Hellmann, Manfred W. (1988): Zwei Gesellschaften - Zwei Sprachkulturen? Acht Thesen zur öffentlichen Sprache in der Bundesrepublik Deutschland und in der Deutschen Demokratischen Republik. In: Goppel, Thomas/von Lojewski, Günther/Eroms, Hans-Werner (Hg.): Wirkung und Wandlung der Sprache in der Politik. Symposium an der Universität Passau [...] vom 25. und 26. November 1988. Passau. S. 89-114.

Kuczynski, Thomas (Hg.) (1995): Das Kommunistische Manifest. Von der Erstausgabe zur Leseausgabe. Mit einem Editionsbericht. Trier. (Schriften aus dem Karl-Marx-Haus 49).

Küpper, Heinz (1966): Wörterbuch der deutschen Umgangssprache. Band 4, Berufsschelten und Verwandtes. Hamburg.

Marzahn, Barbara (1979): Der Deutschlandbegriff der DDR. Düsseldorf. (Schriften des Instituts für deutsche Sprache 48).

Moser, Hugo (1962): Sprachliche Folgen der politischen Teilung Deutschlands. Düsseldorf. (Beihefte zum „Wirkenden Wort" 3).

Moser, Hugo (Hg.) (1964): Das Aueler Protokoll. Deutsche Sprache im Spannungsfeld zwischen West und Ost. Düsseldorf.

Polenz, Peter von (1999): Deutsche Sprachgeschichte. Bd. 3. Berlin/New York.

Riemschneider, Ernst G. (1963): Veränderungen der deutschen Sprache in der sowjetisch besetzten Zone Deutschlands seit 1945. Düsseldorf. (Beihefte zur Zeitschrift „Wirkendes Wort“4). 
Schaeder, Burkhard (2009): Die deutsche Sprache in Ost und West - vor, während und nach der Vereinigung. Rezension zweier Sammelbände [Manfred W. Hellmann/Marianne Schröder (Hg.), Sprache und Kommunikation in Deutschland Ost und West. Ein Reader zu fünfzig Jahren Forschung. Hildesheim 2008, sowie Manfred W. Hellmann, Das einigende Band? Beiträge zum sprachlichen Ost-West-Problem im geteilten und wiedervereinigten Deutschland. Hg. von Dieter Herberg. Tübingen 2008]. In: Muttersprache $1 / 2009$, S. $85-93$.

Schlosser, Horst Dieter (1990): Die deutsche Sprache in der DDR zwischen Stalinismus und Demokratie. Historische, politische und kommunikative Bedingungen. Köln. 2. Auflage 1999.

Schmidt, Hartmut (1997): Real existierende Formelvariation. In: Konerding, Hans-Peter/Lehr, Andrea (Hg.): Linguistische Theorie und lexikographische Praxis. Tübingen. S. 33-50. (Lexicographica, Series Maior 82).

Schmidt, Hartmut (2000a): Hochkomplexe Lexeme. In: Habermann, Mechthild/Müller, Peter O./Naumann, Bernd (Hg.): Wortschatz und Orthographie in Geschichte und Gegenwart. Festschrift für Horst Haider Munske. Tübingen 2000. S. 135-158.

Schmidt, Hartmut (2000b): Entwicklung und Formen des offiziellen Sprachgebrauchs der ehemaligen DDR. In: Besch, Werner/Betten, Anne/Reichmann, Oskar/Sonderegger, Stefan (Hg.): Sprachgeschichte. Ein Handbuch zur Geschichte der deutschen Sprache und ihrer Erforschung. 2., vollständig neu bearbeitete und erweiterte Auflage, 2. Teilband. Berlin/New York. S. 2016-2037.

Schmidt, Hartmut (2000c): Sechzig Jahre und kein bisschen weise. In: Kramer, Undine (Hg.): Lexikologisch-lexikographische Aspekte der deutschen Gegenwartssprache. Tübingen. S. 97-123. (Lexicographica, Series Maior 101).

Schmidt, Hartmut (2001a): Das Reich der Freiheit. In: Burkhardt, Armin/Cherubim, Dieter (Hg.): Sprache im Leben der Zeit. Helmut Henne zum 65. Geburtstag. Tübingen. S. 235-260.

Schmidt, Hartmut (2001b): Ländernamen als Gegenstand der Sprachgeschichte: ,Deutschland' in der Paulskirche. In: Mitteilungen des Deutschen Germanistenverbandes. 48. Jg., Heft 4, S. 556-570.

Schmidt, Hartmut (2002): Traditionen der parlamentarischen Rede. Alte und neue Wörter, Formulierungen und Konstruktionen in den Texten der Frankfurter Nationalversammlung. In: Kramer, Undine (Hg.): Archaismen, Archaisierungsprozesse, Sprachdynamik. Klaus-Dieter Ludwig zum 65. Geburtstag. Frankfurt am Main/Berlin/Bern. S. 55-72.

Schmidt, Hartmut (2004): Artikel 1: „Deutschland ist eine unteilbare demokratische Republik“. Zur gesamtdeutschen Tradition und zum Wandel von Verfassungsformeln in der Geschichte der DDR. In: Reiher, Ruth/Baumann, Antje (Hg.): Vorwärts und nichts vergessen. Sprache in der DDR - Was war, was ist, was bleibt. Berlin. S. 61-73.

Schmidt, Hartmut (2007): „Auferstanden aus Ruinen“. Sprachliche Erbstücke aus schwierigen Zeiten. In: Sprachreport 2007/Heft 3, S. 2-11.

Schmitz-Berning, Cornelia (2000): Vokabular des Nationalsozialismus. Berlin/New York.

Scholz, Heinrich (1964): Einige Beobachtungen zur deutschen Umgangssprache jenseits des Eisernen Vorhangs. In: Moser, Hugo (Hg.): Das Aueler Protokoll. S. 92-101.

WDG (1964-77): Wörterbuch der Deutschen Gegenwartssprache. Hg. von Ruth Klappenbach und Wolfgang Steinitz. Berlin. 6 Bände.

Wigard, Franz (Hg.) (1848-49): Stenographischer Bericht über die Verhandlungen der deutschen constituirenden Nationalversammlung zu Frankfurt am Main. Frankfurt/M. 9 Bde. (durchpaginiert). Dazu: Ders., Vollständiges Inhalts-Verzeichnis (Sach- und Personal-Register). Frankfurt/M. 1850.

Wilss, Wolfram (1989): Anspielungen. Zur Manifestation von Kreativität und Routine in der Sprachverwendung. Tübingen. 
Zemb, Jean Marie (1978): Vergleichende Grammatik Französisch-Deutsch. Comparisation de deux systèmes. Teil 1. Mannheim.

Zentner, Christian/Bedürftig, Friedemann (Hg.) (1993): Das große Lexikon des Dritten Reiches. Augsburg.

\section{Textquellen}

Die angeführten Belege sind mit möglichst genauen Herkunftsangaben versehen, die hier nicht wiederholt werden können. Für ihre Beiträge zur privaten Sammlung habe ich vor allem meiner Kollegin Gabriele Hoppe/Schwetzingen zu danken. Außerdem wurden die großen Textdateien des IDS (erschlossen über COSMAS II) und des Zentrums Sprache der Berlin-Brandenburgischen Akademie der Wissenschaften (DWDS) benutzt (s. die Angaben im Literaturverzeichnis), dazu die Internetsuchmaschine Google sowie Wikipedia. Einige Hörbelege stammen aus Fernsehsendungen der ARD, des ZDF, des ORF1, von 3SAT, aus dem TV-Sender „Das Vierte“ und aus dritten TV-Programmen (HR, SWR).

Stärker abgekürzte Titel von Tages- und Wochenzeitungen sind so aufzulösen:

BERL. ZTG.: Berliner Zeitung

FAZ: Frankfurter Allgemeine Zeitung

MM: Mannheimer Morgen

SZ: Süddeutsche Zeitung

TAZ: tageszeitung [Berlin]

TS: Der Tagesspiegel [Berlin]

Prof. Dr. Hartmut Schmidt 